\title{
Intravenous Transplants of Human Adipose-Derived Stem Cell Protect the Brain from Traumatic Brain Injury-Induced Neurodegeneration and Motor and Cognitive Impairments: Cell Graft Biodistribution and Soluble Factors in Young and Aged Rats
}

\author{
Naoki Tajiri, ${ }^{1,2 \star}$ Sandra A. Acosta, ${ }^{1 *}$ Md Shahaduzzaman, ${ }^{1,3 *}$ Hiroto Ishikawa, ${ }^{1}$ Kazutaka Shinozuka, ${ }^{1}$ Mibel Pabon, ${ }^{1}$ \\ Diana Hernandez-Ontiveros, ${ }^{1,4}$ Dae Won Kim, ${ }^{1}$ Christopher Metcalf, ${ }^{1}$ Meaghan Staples, ${ }^{1}$ Travis Dailey, ${ }^{1}$ \\ Julie Vasconcellos, ${ }^{1}$ Giorgio Franyuti, ${ }^{1}$ Lisa Gould, ${ }^{3,4}$ Niketa Patel, ${ }^{3,5}$ Denise Cooper, ${ }^{3,5}$ Yuji Kaneko, ${ }^{1}$ \\ Cesar V. Borlongan, ${ }^{1,4}$ and Paula C. Bickford ${ }^{1,3,4}$ \\ ${ }^{1}$ Center of Excellence for Aging and Brain Repair, Department of Neurosurgery and Brain Repair and ${ }^{2}$ School of Physical Therapy and Rehabilitation \\ Sciences, University of South Florida Morsani College of Medicine, Tampa, Florida 33612, ${ }^{3}$ James A. Haley Veterans Affairs Hospital, Research Service, \\ Tampa, Florida 33612, and Departments of ${ }^{4}$ Molecular Pharmacology and Physiology and ${ }^{5}$ Molecular Medicine, Morsani College of Medicine, University of \\ South Florida, Tampa, Florida 33612
}

Traumatic brain injury (TBI) survivors exhibit motor and cognitive symptoms from the primary injury that can become aggravated over time because of secondary cell death. In the present in vivo study, we examined the beneficial effects of human adipose-derived stem cells (hADSCs) in a controlled cortical impact model of mild TBI using young (6 months) and aged (20 months) F344 rats. Animals were transplanted intravenously with $4 \times 10^{6}$ hADSCs (Tx), conditioned media (CM), or vehicle (unconditioned media) at $3 \mathrm{~h}$ after TBI. Significant amelioration of motor and cognitive functions was revealed in young, but not aged, Tx and CM groups. Fluorescent imaging in vivo and ex vivo revealed 1,1' dioactadecyl-3-3-3', $3^{\prime}$ tetramethylindotricarbocyanine iodide-labeled hADSCs in peripheral organs and brain after TBI. Spatiotemporal deposition of hADSCs differed between young and aged rats, most notably reduced migration to the aged spleen. Significant reduction in cortical damage and hippocampal cell loss was observed in both Tx and CM groups in young rats, whereas less neuroprotection was detected in the aged rats and mainly in the Tx group but not the CM group. CM harvested from hADSCs with silencing of either NEAT1 (nuclear enriched abundant transcript 1) or MALAT1 (metastasis associated lung adenocarcinoma transcript 1), long noncoding RNAs (lncRNAs) known to play a role in gene expression, lost the efficacy in our model. Altogether, hADSCs are promising therapeutic cellsfor TBI, and IncRNAs in the secretome is an important mechanism of cell therapy.Furthermore, hADSCsshowed reduced efficacy in aged rats, which may in part result from decreased homing of the cells to the spleen.

Key words: aging; brain injury; stem cell

\section{Introduction}

In the United States, an estimated 2 million Americans both young and aged suffer equally from traumatic brain injury (TBI),

Received June 7, 2013; revised 0ct. 22, 2013; accepted Nov. 14, 2013.

Author contributions: L.G., N.P., D.C., C.V.B., and P.C.B. designed research; N.T., S.A.A., M.Sh., H.I., K.S., M.P., D.H.-O., D.W.K., C.M., M.St., T.D., J.V., G.F., Y.K., C.V.B., and P.C.B. performed research; N.T., S.A.A., M.Sh., H.I., L.G., N.P., D.C., Y.K., C.V.B., and P.C.B. analyzed data; N.T., S.A.A., M.Sh., D.C., C.V.B., and P.C.B. wrote the paper.

The authors declare no competing financial interests.

C.V.B. is supported by National Institutes of Health (NIH)/National Institute of Neurological Disorders and Stroke Grant 1R01NS071956-01, Department of Defense Grant W81XWH1110634, James and Esther King Foundation for Biomedical Research Program Grant 1KG01-33966, SanBio, Celgene Cellular Therapeutics, KM Pharmaceutical Consulting LLC and NeuralStem. P.C.B. is supported by Veterans Affairs Medical Research Service, NIH/National Institute on Aging Grants 1P01AG-04418-27 and 1R01 AG044919, and the Michael J. Fox Foundation.

*N.T., S.A.A., and M.Sh. contributed equally to this work.

Correspondence should be addressed to one of the following: Dr. Cesar V. Borlongan, Dr. Paula C. Bickford, Dr. Denise Cooper, or Dr. Yuji Kaneko, Department of Neurosurgery and Brain Repair, University of South Florida, 12901 Bruce B. Downs Boulevard, Tampa, FL 33612. E-mail: cborlong@health.usf.edu; pbickfor@health.usf.edu; Denise.cooper@va.gov; or ykaneko@health.usf.edu.

DOI:10.1523/JNEUROSCI.2425-13.2014

Copyright $\odot 2014$ the authors $\quad 0270-6474 / 14 / 340313-14 \$ 15.00 / 0$ which accounts for $30 \%$ of all injury-related deaths (Faul et al., 2010). However, aging is considered an independent risk factor for negative health outcomes after TBI. In fact, the incidence of TBI-related morbidities and mortally is higher in our aging population and exponentially increases with age (Hawkins et al., 2013).

Recently, stem cell transplantation has been shown to be an effective regenerative therapy for functional and physiological improvement in animal models of brain disorders such as stroke, Parkinson's disease, Alzheimer's disease, and TBI (Lindvall et al., 1990; Clarkson, 2001; Isacson et al., 2001; Bjorklund et al., 2002; Mahmood et al., 2003; Yang et al., 2003; Fraser et al., 2006; Bjugstad et al., 2008; Muraoka et al., 2008; Harting et al., 2009; Liu et al., 2009). An attractive cell therapy for regenerative medicine and wound healing is the use of mesenchymal stem cells (MSCs) derived from adipose tissue and their secretome. Human adiposederived stem cells (hADSCs) have the potential to proliferate, differentiate into different cell lineages, secrete an extensive se- 
cretome containing growth factors, cytokines, chemokines, microRNAs, and long noncoding RNA (lncRNA), and have therapeutic potential in TBI (Lendeckel et al., 2004; Xue et al., 2010). Of the many lncRNAs that are secreted, two are important for cellular differentiation, nuclear enriched abundant transcript 1 (NEAT1) and metastasis associated lung adenocarcinoma transcript 1 (MALAT1), because of their ability to assist in the alternative splicing of numerous pre-mRNA (Wapinski and Chang, 2011; Derrien et al., 2012; Zhang et al., 2012). Stem cells, which are in a proliferative, nondifferentiating state, secrete numerous lncRNA, including NEAT1 and MALAT1. Stem cells, which are not differentiating, are apparently shedding the factors that would assist in differentiation until appropriate cues are in place. However, the secretion of lncRNA does not exclude lncRNA uptake by adjacent cells, which could influence survival and regeneration of these cells by undisclosed modalities, including mRNA splicing, migration, and regulation of gene expression (Kim et al., 2007; Ikegame et al., 2011; Sun et al., 2012). The majority of cell transplantation studies are performed in young animals; in fact, we now understand that not only is endogenous neurogenesis affected in aging (Conboy et al., 2005; Carlson and Conboy, 2007), a few reports suggest that the transplanted stem cells do not survive as well in the aged host (Conboy et al., 2005; Carlson and Conboy, 2007). However, less well studied is the mechanism of action of stem cells and if there may be alterations in migration of cells to various peripheral organs. In the present study, we evaluated the effects of intravenous administration of hADSCs on motor and cognitive function and their homing pattern during acute and subacute periods in young and aged TBI rats with particular emphasis on the spleen, which has been implicated as a major source of systemic inflammation (Vendrame et al., 2006) and is known to be necessary for the neuroprotective action of MSCs after TBI pathology (Walker et al., 2009, 2010). To elucidate hADSC mechanism of action, we examined conditioned media (CM) from hADSCs grown with antisense RNA to silence two lncRNAs known to play roles in cell survival, inflammation, and gene expression.

\section{Materials and Methods}

Subjects. Experimental procedures were approved by the University of South Florida Institutional Animal Care and Use Committee (IACUC). All animals were housed under normal conditions $\left(20^{\circ} \mathrm{C}, 50 \%\right.$ relative humidity, and a $12 \mathrm{~h} \mathrm{light/dark} \mathrm{cycle).} \mathrm{All} \mathrm{studies} \mathrm{were} \mathrm{performed} \mathrm{by}$ personnel blinded to the treatment condition.

Fluorescent labeling of cultured hADSC grafts and CM preparation. hADSCs were obtained from ZenBio (catalog \#ASC-S). According to the protocol of the manufacturer, cells $\left(6.7 \times 10^{5}\right.$ cells/T75 flask) were suspended in $10 \mathrm{ml}$ of supplemented growth medium (PM-1; ZenBio) and grown in noncoated T-75 flasks at $37^{\circ} \mathrm{C}$ in humidified atmosphere containing $5 \%$ carbon dioxide. hADSCs were grown until they were $90 \%$ confluent and then subcultured. Cells were routinely assessed by flow cytometry for stem cell markers and were verified as CD31 ${ }^{-}, \mathrm{CD} 34^{-}$, $\mathrm{CD} 45^{-}, \mathrm{CD} 106^{-}, \mathrm{CD}_{117^{-}}$and CD44 90\% ${ }^{+}, \mathrm{CD} 7389 \%^{+}, \mathrm{CD} 105$ $80 \%{ }^{+}, \mathrm{CD} 9097 \%{ }^{+}$. Thus, three major markers that are routinely used to define MSCs, CD105, CD73, and CD90, are present and endothelial or hematopoietic markers are absent. This profile remained stable for 10 passages; all cells used in this study for transplantation or generation of $\mathrm{CM}$ were between two and nine passages. Cells were also routinely assessed for multipotency using methods as published previously. Differentiation into osteoblasts was performed by culturing cells in osteoblast differentiation medium (DM; ZenBio) for 4 weeks with changes every 5 d. Positive staining for Alizarin Red (1\% Alizarin Red; CM-0058; Lifeline Technology) was used to verify osteoblast differentiation. Cells were fixed in PBS-10\% Formalin for 20 min, washed once with PBS, stained with $1 \%$ Alizaren Red $\mathrm{S}$ for $5 \mathrm{~min}$, washed again three times, and visual- ized in a light microscope. Differentiation to adipocytes was performed using DM2 adipocyte DM (ZenBio) for $12 \mathrm{~d}$ with medium changes every $5 \mathrm{~d}$. Oil Red was used to identify adipocytes. Cells were fixed in PBS-10\% Formalin for $1 \mathrm{~h}$, washed in isopropanol, and dried. Oil Red O working solution was added for $10 \mathrm{~min}$ and then washed four times with PBS. Cells were imaged using a light microscope. hADSCs were able to differentiate into both osteoblasts and adipocytes. For graft preparation, hADSCs were harvested, and the cell density was adjusted as $4 \times 10^{6}$ cells in $500 \mu \mathrm{l}$ of PBS. Then cells were incubated with XenoLight $1,1^{\prime}$ dioctadecyl-3,3,3',3'-tetramethylindotricbocyanine iodide (DiR) (cata$\log \# 125964$; Caliper Life Sciences) for $30 \mathrm{~min}$ to evaluate migration of the transplanted hADSCs. After the labeling, cells were rinsed using PBS and centrifuged twice. Thereafter, the pellet of labeled cells was suspended in $500 \mu \mathrm{l}$ of PBS just before the transplantation. In each passaging, CM was collected from hADSC culture, filtered using $0.45 \mathrm{~mm}$ poresized filter to avoid cells contamination, and cryopreserved for additional experiments. For the present study, we used the CM from cells at passages 2 to 4.

Preparation of hADSC-derived CM with knockdown of NEAT1 and MALAT1. The cultured hADSCs at $80 \%$ confluence were treated with antisense RNA (NEAT1 and MALAT1 or scramble control obtained from ISIS Pharmaceuticals) that was incorporated into the cells for $48 \mathrm{~h}$, and $\mathrm{CM}$ was collected for $24 \mathrm{~h}$ and stored at $-80^{\circ} \mathrm{C}$ until needed. $\mathrm{CM}$ was verified to be deficient of either NEAT1 or MALAT1 in the appropriate conditions, and no change in either was observed with the scrambled antisense RNA control.

Measurement of human vascular endothelial growth factor, stem cell factor, and tissue inhibitor of metalloproteinase 3 concentration. Cell supernatant was measured by Human VEGF Quantikine ELISA Kit (DVE00; R\&D Systems), Human SCF Quantikine ELISA Kit (DCK00; R\&D Systems), and Human TIMP-3 DuoSet (DY973; R\&D Systems), according to the instructions of the manufacturer. Absorbance from each sample was measured using a Synergy HT plate reader (Bio-Tex) at $450 \mathrm{~nm}$.

Surgical procedures. A total of 82 Fisher 344 male rats, young (6 months, $n=48$ ) and aged (20 months; $n=34)$, were subjected to either TBI using the controlled cortical impact (CCI) injury model (Pittsburgh Precision Instruments) or sham control (no TBI). The total number of rats in each group was as follows: $n=9$ for young TBI-hADSC; $n=8$ for young TBI-CM; $n=6$ for young TBI-CM knockdown (kd) NEAT1; $n=$ 6 for young TBI-CM kdMALAT $1 ; n=4$ for young TBI-CM kdscramble; $n=7$ for young TBI-vehicle; $n=8$ for young sham control; $n=11$ for aged TBI-hADSC; $n=8$ for aged TBI-CM; $n=8$ for aged TBI-vehicle; and $n=7$ for aged sham control. Deep anesthesia was achieved using $1-2 \%$ isoflurane, and it was maintained using a gas mask. All animals were fixed in a stereotaxic frame (David Kopf Instruments). After exposing the skull, coordinates of $+0.2 \mathrm{~mm}$ anterior and $+0.2 \mathrm{~mm}$ lateral to the midline (Paxinos and Watson, 2007) were used and impacted the brain at the frontoparietal cortex with a velocity of $6.0 \mathrm{~m} / \mathrm{s}$, reaching a depth of $0.5 \mathrm{~mm}$ (mild TBI) below the dura matter layer and remaining in the brain for $150 \mathrm{~ms}$. The impactor rod was angled $15^{\circ}$ vertically to maintain a perpendicular position in reference to the tangential plane of the brain curvature at the impact surface. A linear variable displacement transducer (Macrosensors) that was connected to the impactor measured the velocity and duration to verify consistency. Sham control injury surgeries (i.e., uninjured animals) consisted of animals exposed to anesthesia, scalp incision, craniectomy, and suturing. An electric drill was used to performed the craniectomy of $\sim 4 \mathrm{~mm}$ radius centered from bregma +0.2 anterior and $+0.2 \mathrm{~mm}$ lateral right (Paxinos and Watson, 2007). A computer-operated thermal blanket pad and a rectal thermometer allowed maintenance of body temperature within normal limits. All animals were closely monitored postoperatively with weight and health surveillance recording as per IACUC guidelines. Rats were kept hydrated at all times, and the analgesic ketoprofen was administered after TBI surgery and as needed thereafter. Before and after TBI, rats were fed regular rodent diet. A schematic diagram of the experimental design is shown (Fig. 1).

Intravenous administration of hADSCs, CM, and vehicle. Three hours after TBI surgery, rats were anesthetized with $1-2 \%$ isoflurane in nitrous oxide/oxygen $(69 \% / 30 \%)$ using a face mask. Vehicle (unconditioned 
Experiment \#1

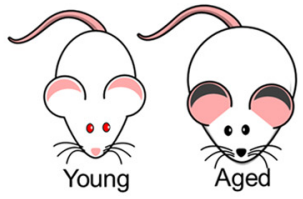

Day 0: Baseline behavioral testing (EBST, forelimb akinesia test, and paw grasp test)

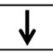

Day 0: TBI

$\downarrow$

Day 0: Transplantation of hADSC, CM, or vehicle at 3hrs post-TBI

$\downarrow$

Day 1, 3, 7: EBST, forelimb akinesia test, paw grasp test

$\downarrow$

Day 7-11: RAWM test

$\downarrow$

Day 11: Euthanasia for histological evaluation

\section{Experiment \#2}

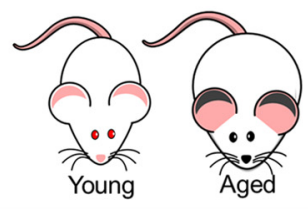

Day 0: Transplantation of fluorescent DiR-hADSC, CM, or vehicle at 3hrs post-TBI $\downarrow$

Day 0: $1 \mathrm{hr}, 4 \mathrm{hrs}, 12 \mathrm{hrs}, 24 \mathrm{hrs}, 72 \mathrm{hrs}$, Day 11 IVIS Fluorescent Imaging (near infra-red)

\section{Experiment \#3}
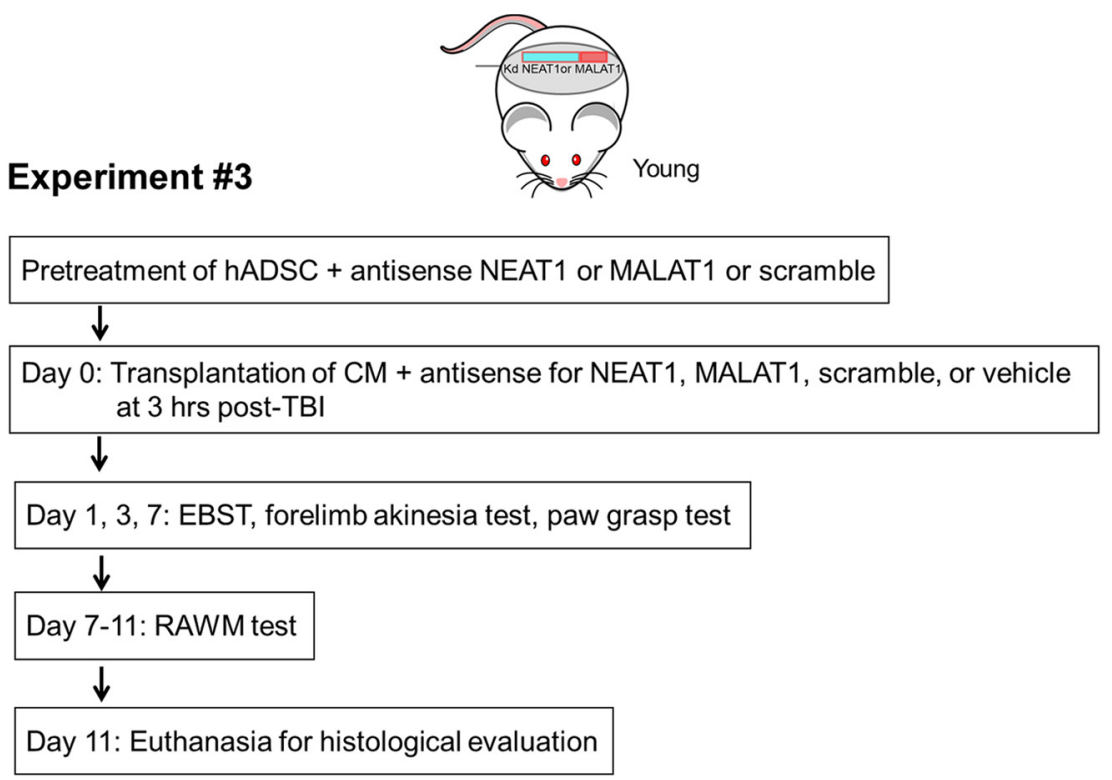

Figure 1. Experimental design. Experiment \#1, Motor behavioral testing, EBST, forelimb akinesia, and paw-grasp test were performed in young and aged rats before TBI. Young and aged rats were subjected to mild TBI and received transplants of hADSCS, CM, or vehicle $3 \mathrm{~h}$ after TBI. After motor and cognitive behavioral evaluations, at days 1, 3, and 7, all rats were killed for immunohistochemical evaluations. Experiment \#2, Young and aged rats received transplants of DiR fluorescent labeled $\mathrm{hADSC}, \mathrm{CM}$, or vehicle at $3 \mathrm{~h}$ after TBI. IVIS fluorescent imaging was done at 1, 4, 12, 24, and $72 \mathrm{~h}$ and day 11 after transplantation. Experiment \#3, hADSCs were pretreated with antisense for NEAT1 or MALAT1 or scramble. CM from these cells was collected and infused 3 hafter TBI. Motor and cognitive behavioral tests were performed as in Experiment 1 . After $11 \mathrm{~d}$ after CM infusion, all rats were killed for histological evaluations. media; $500 \mu \mathrm{l}$ of sterile media PM-1 that was not incubated with cells), CM (500 $\mu$ l of CM, CM kdNEAT1, CM kdMALAT1, CM kdscramble), or hADSC grafts $\left(4 \times 10^{6}\right.$ viable cells in $500 \mu \mathrm{l}$ of sterile saline) were administered via the jugular vein.

XenoLight DiR for in vivo and ex vivo biodistribution imaging procedures. Young and aged rats were subjected to TBI using the CCI injury model (Pittsburgh Precision Instruments). DiR-labeled $4 \times 10^{6} \mathrm{hADSCs}$ (Tx group) were then transplanted into the jugular vein at $3 \mathrm{~h}$ after TBI. To visualize DiR fluorescence emitted from the engrafted hADSCs in vivo, animals were shaved to avoid light scattering and anesthetized in a chamber with $3.0 \%$ isoflurane. Once the rats were completely anesthetized, the animals were transferred from the chamber to the IVIS Spectrum 200 Imaging System (Xenogen), and the isoflurane level was set at $1-2 \%$ until complete image acquisition. The biodistribution of DiR-labeled hADSC grafts was monitored at 1, 4, 12, 24, 48, and $72 \mathrm{~h}$ after transplant. Rats were imaged ventrally at all time points. A second set of images were obtained for the head region using a higher magnification. Identical illumination parameters [exposure time $=$ Auto; lamp voltage $=$ high; $\mathrm{f} /$ stop $=2$; field of view $=\mathrm{B}$ (for head) and $\mathrm{C}$ (for whole body); binning $=8$; emission filter $=800 \mathrm{~nm}$; and excitation filter $=745$ $\mathrm{nm}$ ] were selected for each acquisition. All captured images were analyzed with Living Image software 4.0 (Xenogen). To analyze the change in DiR fluorescence intensity, identical regions of interest (ROIs) were placed on the abdomen and head area for animals. The same ROI was also placed on the control animal as the background reference. Background efficiency was subtracted from each of the individual animal's efficiency and presented as an average radiant efficiency (photons per second per square centimeter per steridian divided by microwatts per square centimeter).

Behavioral testing. Each rat was subjected to a series of behavioral tests to reveal motor, neurological, and cognitive performance of animals, before and after TBI and after transplantation. The tests included the elevated body swing test (EBST), forelimb akinesia, and paw-grasp test before and after TBI at days 0,1 , 3 , and 7 . The radial arm water maze (RAWM) test was performed on day 7 after TBI surgery.

EBST test. EBST is a measure of asymmetrical motor behavior that does not require animal training or drug injection (Borlongan and Sanberg, 1995). The rats were held, in the vertical axis, $\sim 1$ inch from the base of its tail and then elevated to an inch above the surface on which it has been resting. The frequency and direction of the swing behavior were recorded for 20 tail elevations. A swing was counted when the head of the rat moved $>10^{\circ}$ from the vertical axis to either side. The total number of swings made to the biased side was added per group and divided by the $n$, giving us the average number of swings per treatment group.

Forelimb akinesia test. Before and after TBI surgery, young and aged rats from sham con- 
trol, TBI-vehicle, TBI-hADSC, or TBI-CM were evaluated for forelimb akinesia (Borlongan et al., 1998). Ipsilateral and contralateral forepaw strength and motility were scored by two experimentally blinded evaluators using the following forelimb akinesia scale. On a scale of 1 to 3,1 is normal, 2 is impaired, and 3 is severely impaired. Scores were tallied for each individual animal, and then mean scores for treatment groups were used for analyses.

Paw-grasp test. Before and after TBI surgery, grip strength of young and aged rats from sham control, TBI-vehicle, TBI-hADSC, or TBI-CM were evaluated. An abnormal grip is indicative of impaired neuromuscular function. In this test, rats were held by their bodies against a pole (Ibrahim et al., 2009). Both ipsilateral and contralateral paw grip strength were scored by two experimentally masked evaluators using the following grip strength scale. In a scale of 1 to 3,1 is normal, 2 is impaired, and 3 is severely impaired. Scores were tallied for each individual animal, and then mean scores for treatment groups were used for analyses.

RAWM test. The RAWM test allows the investigator to analyze basic parameters, such as place or spatial learning. The acquisition of learning is when the animal must learn how to use distal cues to navigate through the arms to find the hidden platform. Also, it has been shown that RAWM spatial learning is not dependent on locomotor ability, because this does not affect swimming speed (Vorhees and Williams, 2006). Each error shows the ability to search and learn to use distal cues to find the platform. To reveal the cognitive effects of hADSC grafts on spatial navigation and memory of young and aged TBI rats, RAWM, a hippocampal-dependent task, was tested. RAWM started on day 7 after TBI surgery and intravenous transplantation of hADSC grafts and CM. Six-arm RAWM was placed in a water tank of $\sim 150 \mathrm{~cm}$ diameter, and a 40 -cm-height, 10 -cm-diameter platform was used. The platform was submerged $1 \mathrm{~cm}$ below the surface of water. The temperature of the water was kept at $27^{\circ} \mathrm{C}$. Rats were placed on the start arm at the beginning of every trial, and the platform was located on the goal arm. Every animal had an assigned platform/arm location throughout acquisition of learning, yet the starting zone was randomly changed per trial. A spacetraining protocol was followed. Rats were given two blocks of four trials, with each block separated by $30 \mathrm{~min}$ rest period per day, for a total of eight trials a day for $3 \mathrm{~d}$ of acquisition of learning. Trials were only $60 \mathrm{~s}$ long. Once animals found their goal arm/platform, they were allowed to remain on the platform for $30 \mathrm{~s}$ between trials. If rats were unable to find their goal arm/platform within $60 \mathrm{~s}$, rats were guided to their goal arm and allowed to rest on the platform for $30 \mathrm{~s}$. On day 4, a probe trial was given $1 \mathrm{~h}$ before reversal training started, placing the rat $180^{\circ}$ from the goal arm. Rats were given four trials to train for the new position (reversal training). RAMW performance analysis was done by averaging the trials per block, using four trials per block and then a total of two blocks per day (errors are given every time rats are not entering the goal arm). Reversal training was analyzed by counting the total of errors in each trial.

Brain and organ harvesting, fixation, and sectioning. Under deep anesthesia, rats were killed on day 11 after TBI for immunohistochemical investigations. Briefly, animals were perfused through the ascending aorta with $200 \mathrm{ml}$ of cold PBS, followed by $200 \mathrm{ml}$ of $4 \%$ paraformaldehyde in phosphate buffer (PB). Brains, spleen, lungs, and liver were removed and postfixed in the same fixative for $24 \mathrm{~h}$, followed by $30 \%$ sucrose in PB until completely sunk. Six series of coronal sections were cut at a thickness of $30 \mu \mathrm{m}$ with a cryostat and stored at $-20^{\circ} \mathrm{C}$.

Measurement of impact and peri-impact area: Nissl staining analysis. Serial sections corresponding to the same group of animals were stained with Nissl for impact- and peri-impact calculations. Six coronal sections between the anterior edge and posterior edge of the impacted area were collected and processed for Nissl staining from each brain perfused at day 11 after TBI. Sections were cut at a thickness of $30 \mu \mathrm{m}$ by a cryostat. Every sixth coronal tissue section, beginning at anteroposterior (AP) +2.28 $\mathrm{mm}$ and ending at AP $0 \mathrm{~mm}$ posterior from bregma (Paxinos and Watson, 2007), was randomly selected for measurement of impact- and periimpact area. Brain sections were examined using a light microscope (Olympus) and Keyence microscope. The impact area of brain damage was measured in each slice and quantified by a computer-assisted image analysis system (NIH Image) and calculated by the following formula: $[($ area of the damaged region in each section) $\times 0.030]$ (cubic millime- ters). The peri-impact area of brain damage was counted by a computerassisted image analysis system (NIH Image). Impact and peri-impact area was then expressed as a percentage of the ipsilateral hemisphere compared with the contralateral hemisphere.

Measurement of hippocampal cell loss: hematoxylin and eosin staining analysis. Hematoxylin and eosin (H\&E) staining was performed to confirm the core impact injury of our TBI model. As shown in our previous studies (Hayashi et al., 2009; Yu et al., 2009; Glover et al., 2012; Acosta et al., 2013), we demonstrated primary damage to the frontoparietal cortex. $\mathrm{H} \& \mathrm{E}$ staining was also analyzed in the hippocampal area. Starting at coordinates AP $-1.7 \mathrm{~mm}$ and ending AP $-3.9 \mathrm{~mm}$ from bregma (Paxinos and Watson, 2007), coronal brain sections $(30 \mu \mathrm{m})$ covering the whole dorsal hippocampus. A total number of six sections per rat were used. Cells presenting with nuclear and cytoplasmic staining (H\&E) were manually counted in the CA3 neurons. CA3 cell counting spanned the whole CA3 area, starting from the end of hilar neurons to the beginning of curvature of the CA2 region in both the ipsilateral and contralateral sides. Sections were examined with Nikon Eclipse 600 microscope at $20 \times$. All data are represented as mean \pm SEM values, with statistical significance set at $p<0.05$.

Measurement of cell survival: human nuclei staining analysis. Every sixth $30-\mu \mathrm{m}$-thick coronal tissue section of brain and spleen, spanning the area of injury in the case of the brain and the entire red pulp in the case of spleen were randomly selected for quantitative analysis. Free-floating sections were washed three times for $5 \mathrm{~min}$ in PBS. For human nuclei $(\mathrm{HuNu})$ staining, samples were blocked for $60 \mathrm{~min}$ at room temperature with 5\% normal goat serum (Invitrogen) in PBS containing 0.1\% Tween 20 (PBST; Sigma). Sections were then incubated overnight at $4^{\circ} \mathrm{C}$ with mouse monoclonal anti-HuNu (1:50; MAB1281; Millipore) with 5\% normal goat serum. The sections were washed five times for $10 \mathrm{~min}$ in PBST and then soaked in 5\% normal goat serum in PBST containing corresponding secondary antibodies, goat anti-mouse IgG Alexa Fluor 488 (green; 1:500; Invitrogen), for 90 min. Finally, sections were washed five times for $10 \mathrm{~min}$ in PBST and three times for $5 \mathrm{~min}$ in PBS, then processed for Hoechst 33258 (bisBenzimideH 33258 trihydrochloride; Sigma) for $30 \mathrm{~min}$, washed in PBS, and coverslipped with Fluoromount (Sigma). Brain and spleen sections were examined using a confocal microscope (Olympus). Control studies included exclusion of primary antibody substituted with $5 \%$ normal goat serum in PBS. No immunoreactivity was observed in these controls.

Flow cytometry. Immunophenotypical analysis of cultured cells was performed using FITC-, phycoerythrin-, or adenomatous polyposis coliconjugated monoclonal antibodies against CD31, CD34, CD44, CD45, CD73, CD90, CD105, CD106, and CD117 and appropriate isotype controls. Cells were detached using TryLE Select (Invitrogen), washed, and resuspended at a concentration of $10^{6}$ cells $/ \mathrm{ml}$. Cells were incubated at $4^{\circ} \mathrm{C}$ for $10 \mathrm{~min}$ in PBS with 10\% FBS. Cells were centrifuged for $5 \mathrm{~min}$ at $1200 \mathrm{rpm}$. The cell pellet was resuspended in the binding buffer (PBS/2\% FBS/ $0.01 \%$ sodium azide), followed by incubation with optimized concentrations of specific mAbs at $4^{\circ} \mathrm{C}$ for $30 \mathrm{~min}$, then washed with the binding buffer, resuspended in $0.5 \mathrm{ml}$ of the same buffer, and analyzed within $1 \mathrm{~h}$ using the BD Accuri C6 flow cytometer (BD Biosciences).

Statistical analyses. The data were evaluated statistically using either two-way ANOVA or repeated-measures ANOVA and subsequent post hoc Scheffé's or Bonferroni's test for behavior. Statistical significance was preset at $p<0.05$.

\section{Results}

To test the hypothesis that administration of hADSC grafts can rescue motor impairments associated with TBI in young and aged rats, a battery of motor behavioral tests, EBST, forelimb akinesia, and paw-grasp tests were conducted.

\section{hADSC grafts and CM improve TBI-associated motor asymmetry in young, but not aged, TBI rats}

EBST revealed that young and old rats subjected to TBI-vehicle exhibited significant asymmetry in motor activity at days 1,3 , and 7 compared with sham control rats (two-way ANOVA, $F_{(3,12)}=$ 


\section{Motor assesment}

A
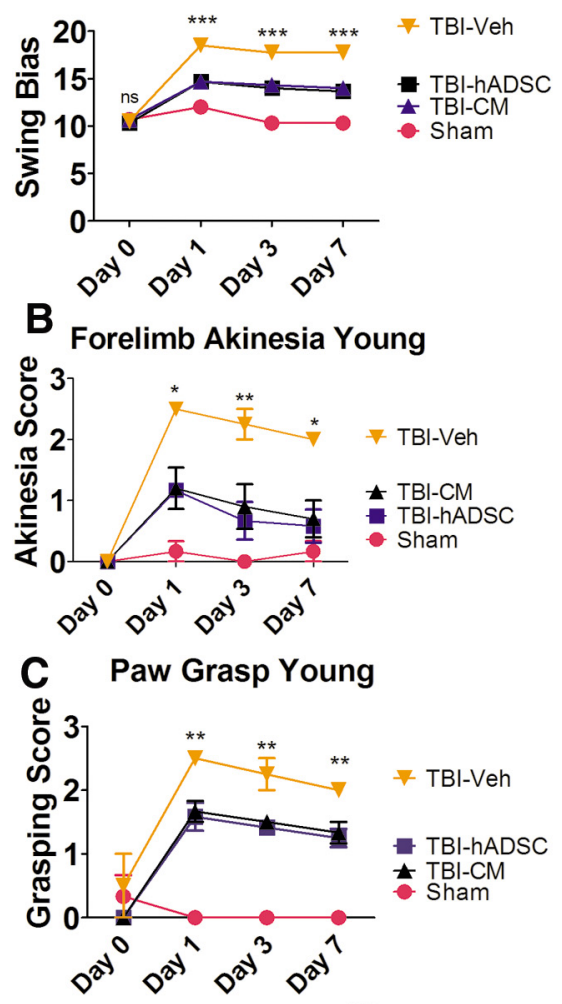
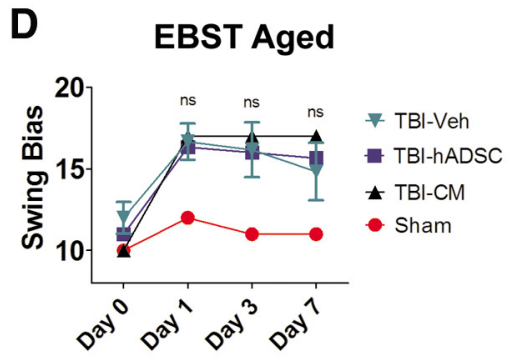

E Forelimb Akinesia Aged

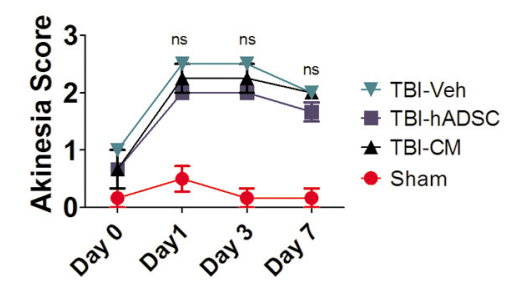

$\mathbf{F}$

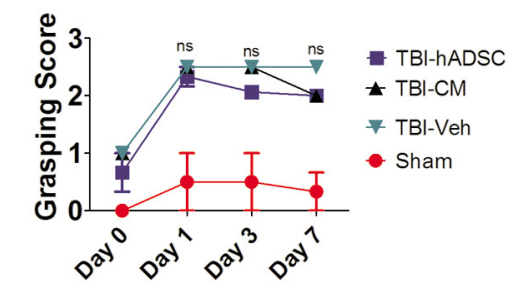

hADSC grafts and CM ameliorate TBIassociated forelimb akinesia in young, but not aged, TBI rats

The forelimb akinesia test demonstrated TBI-associated impairment in forelimb strength in young and aged rats treated with vehicle at all time points compared with sham control rats (two-way ANOVA, $F_{(3,12)}=12.06, p<0.001$; Fig. $\left.2 B, E\right)$. hADSC grafts and CM significantly improved forelimb movement and function in young rats exposed to TBI within the first $24 \mathrm{~h}$, which continued throughout testing period relative to young rats exposed to TBI treated with vehicle and sham controls $(p<0.05$; Fig. $2 B)$. Aged animals subjected to TBI did not display any amelioration of the forelimb akinesia after treatment with either hADSCs or CM relative to aged TBI-vehicle and sham control ( $p>0.05$; Fig. $2 E)$. All data are represented as mean \pm SEM values.

\section{hADSC grafts and CM attenuate TBI- associated paw-grasp impairment in young, but not aged, TBI rats}

Assessment of paw-grasp function demonstrated TBI-associated impairments in paw grasp in both young and aged rats subjected to TBI treated with vehicle at all time points compared with sham control rats (two-way ANOVA, $F_{(3,12)}=31.80$, $p<0.001$; Fig. 2C,F). At $24 \mathrm{~h}$ after the onset of TBI, young rats treated with either hADSC grafts or CM scored significantly better on the paw-grasp test compared with young rats exposed to TBI treated with vehicle and sham controls $(p<0.05$; Fig. $2 C)$. There was no significant treatment effect in the paw-grasp assessment of aged rats subjected to TBI treated with hADSCs or CM relative to aged TBI-vehicle and sham controls ( $p>$ 0.05 ; Fig. $2 F$ ). All data are represented as mean \pm SEM values.

Figure 2. Motor assessment. Results indicate that transplantation of hADSCs or infusion of CM rescued the TBIassociated motor deficits in young rats only. Asterisks denote significant difference in motor impairments in young rats transplanted with hADSCs or infused with CM relative to TBI-vehicle group and sham controls in EBST $(\boldsymbol{A})$, forelimb akinesia $(\boldsymbol{B})$, and paw-grasp test $(\boldsymbol{C})$. Two-way ANOVA revealed significant treatment effects as follows: $\mathrm{EBST}, \boldsymbol{F}_{(3,12)}=24.66, p<$ 0.0001; Bonferroni's test, ${ }^{* * *} p<0.001$; forelimb akinesia, $F_{(3,12)}=16.55, p<0.0001$; Bonferroni's test; ${ }^{*} p<0.05$; paw-grasp test, $F_{(3,12)}=31.80, p<0.0001$; Bonferroni's test, ${ }^{* *} p<0.01$. $\boldsymbol{D}-\boldsymbol{F}$ show that there is no significant treatment effects on the motor behavior in aged rats (Bonferroni's test, $p>0.05$ ). $\mathbf{G}$ shows a significant correlation between peri-infarct area and EBST motor performance. Data shown are for the young and aged analyzed separately, but the significance remains when the groups are combined $\left(R^{2}\right.$ for young $=0.93$; aged $=0.81$; combined $=0.83$; Pearson's $r$ for young $=-0.97$; aged $=-0.91$; combined $=-0.91)$.

24.66, $p<0.001$; Fig. 2). Significant recovery of motor symmetry was detected in the young rats treated with either hADSCs or CM compared with young rats subjected to TBI-vehicle and sham controls ( $p<0.05 ;$ Fig. $2 A)$. The effect of cell therapy and CM to improve motor asymmetry in young rats exposed to TBI was observed as early as $1 \mathrm{~d}$ after treatment and continued throughout the testing period ( $p<0.05$; Fig. $2 A$ ). Unlike the recovery observed in young rats, aged animals did not display any treatment effect with either hADSC grafts or CM relative to aged rats treated with vehicle and aged sham controls ( $p>0.05$; Fig. $2 D)$.
hADSC grafts and CM ameliorate TBIassociated cognitive impairments found in RAWM in young animals but showed reduced effects in aged animals

Young and aged sham controls, subjected to TBI treated with vehicle (unconditioned media), hADSC grafts, or $\mathrm{CM}$, were tested for spatial memory using the six-arm spaced training RAWM task to evaluate their cognitive function. In this behavioral paradigm, the rats were trained for four trials a day for 3 consecutive days to find the hidden escape platform. During the acquisition of learning, there were no differences in performance demonstrating that all groups of rats, young and aged, were able to learn the location of the hidden platform ( $p>0.05$; Fig. $3 A, C)$. Of note, there were no disparities of motor function and swimming capabilities, indicating that 


\section{A Radial Arm Water Maze Young}

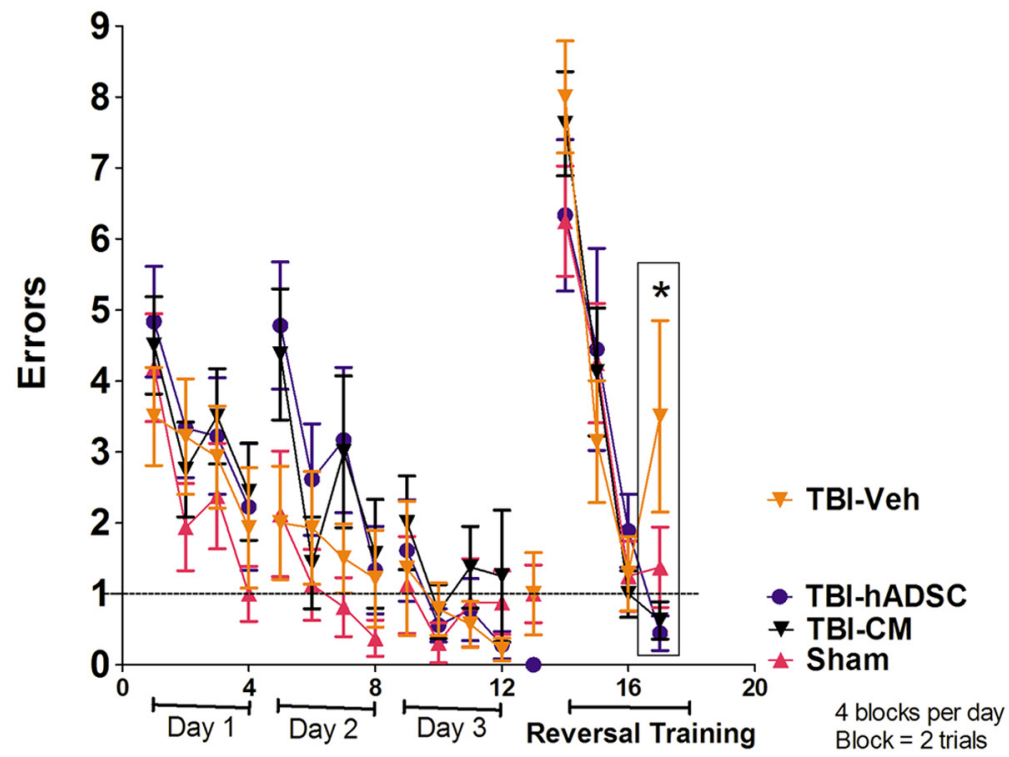

\section{Radial Arm Water Maze}

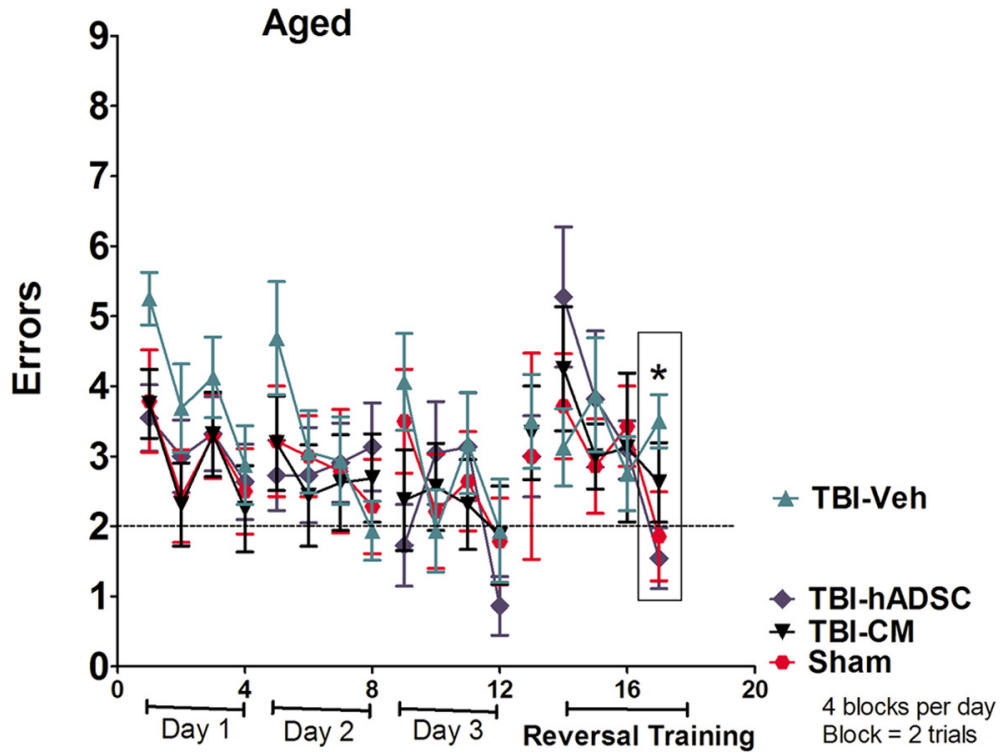

\section{B Young Rats Reversal Training Trial \#4}

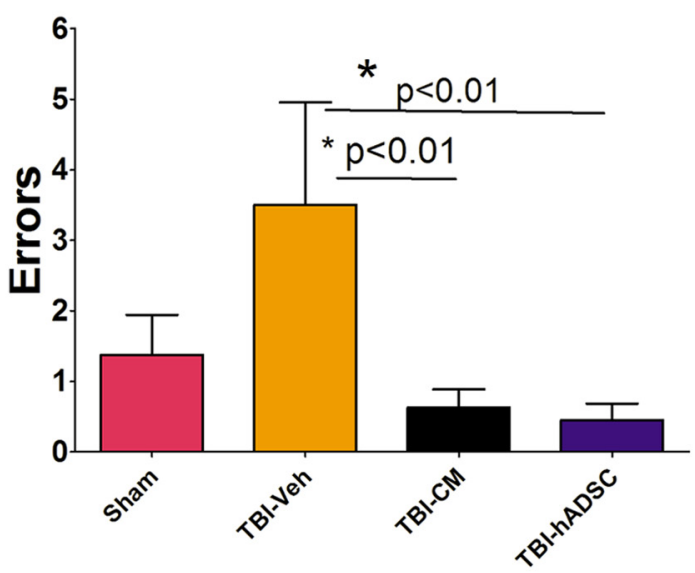

D Aged Rats Reversal Training Trial \#4

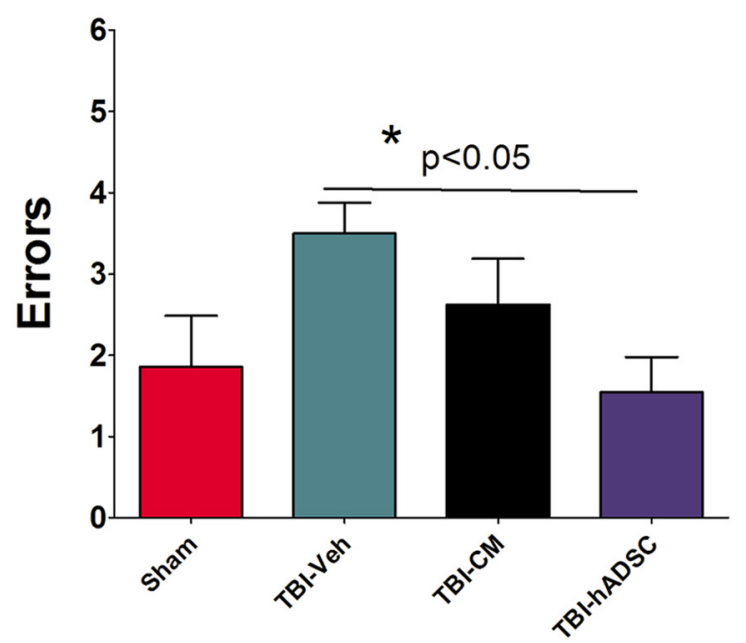

Figure 3. Cognitive assessment. Results indicate that cognitive deficits associated with TBI were ameliorated after transplantation of hADSCs in both young and aged rats in reversal training relative to vehicle and sham control. One-way ANOVA revealed significant treatment effects in the young $\left(F_{(3,28)}=3.95, p<0.0185 ;\right.$ Bonferroni's test, $\left.p<0.05\right)$ and aged $\left(F_{(3,28)}=3.149, p<\right.$ 0.01 ; Bonferroni's test, $\left.{ }^{*} p<0.05\right)$ rats. CM treatment decrease the cognitive-associated TBl impairments only in young rats relative to vehicle and sham controls $(p<0.05)$. $A$ and $C$ shows escape errors (mean \pm SEM) of young and aged rats, respectively, to find the hidden platform in the RAWM for days 1-3 (4 blocks per day; a block is 2 trials) and for reversal testing on day 4 (trials $1-4$ ). $\boldsymbol{B}$ and $\boldsymbol{D}$ show trial 4 from the reversal training for young and aged rats, respectively.

they were equally able to escape from the water by finding the platform during the allotted $60 \mathrm{~s}$ learning trials. hADSC grafts or CM significantly ameliorated the TBI-associated cognitive impairment in young rats relative to young rats treated with vehicle and sham controls (one-way ANOVA, $F_{(3,28)}=22.98$, $p<0.001$; Fig. 3B). There were significant treatment effects on cognitive function in aged rats treated with hADSCs but not with $\mathrm{CM}$ relative to aged rats treated with vehicle and sham controls $(p>0.05$; Fig. $3 D)$. All data are represented as mean \pm SEM values.
Age-dependent biodistribution and homing of systemically administrated hADSCs in vivo

hADSCs were labeled in vitro with XenoLight and administered through the jugular vein of young and aged rats $3 \mathrm{~h}$ after TBI, and their biodistribution was tracked at $1,4,12,24,48$, and $72 \mathrm{~h}$ (Fig. $4 A-C)$. Of note, to show that the signal that we detected from the transplanted group was specific for the DiR signal, young and aged sham control rats were subjected to the IVIS imager, and, as expected, there was no fluorescent signal detected. Within the first hour after transplantation, the spleen and liver area of young 
A Young and aged rats in vivo bio-distribution

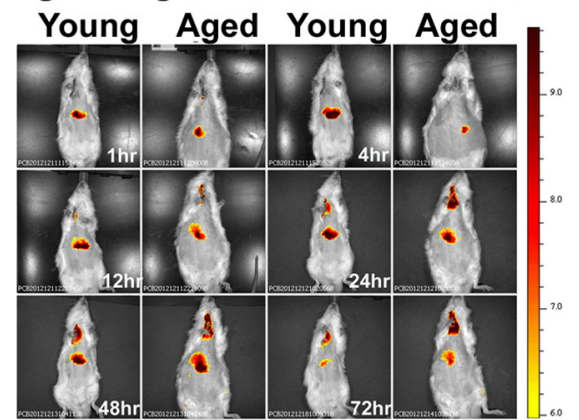

Radiant Efficien

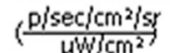

Color Scale Min $=5.97 \mathrm{e}$

$\operatorname{Max}=9.63 \mathrm{e} 7$

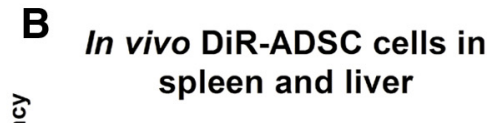

C In vivo DiR-ADSC cells in Head
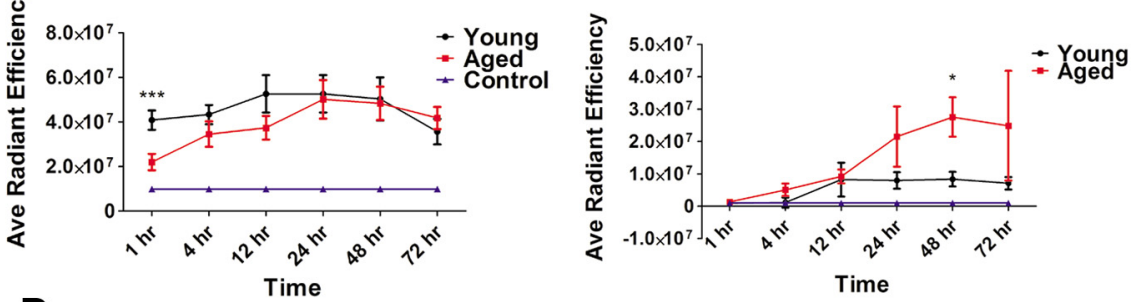

D Young and aged rats ex vivo bio-distribution

Brain
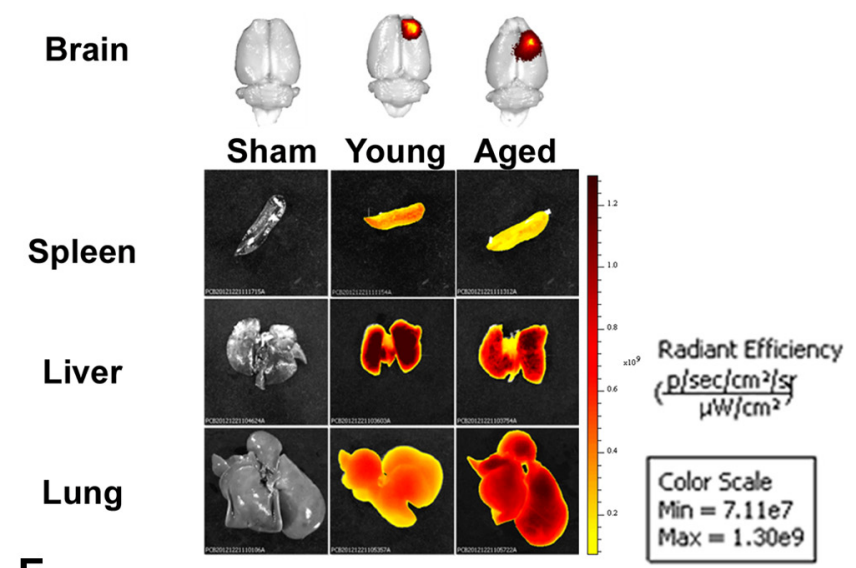

\section{E DiR-hADSC cells in organs}

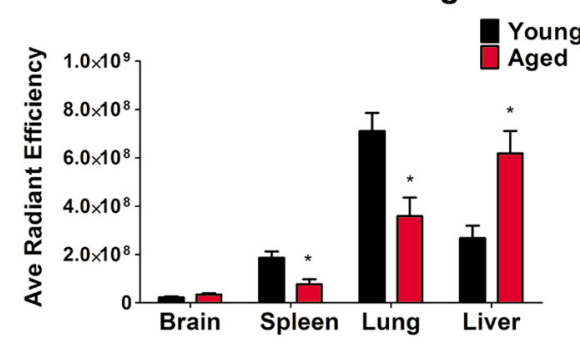

Figure 4. Biodistribution of DiR-labeled hADSCS after TBI. In vivo near-infrared IVIS imaging revealed that the biodistribution and homing of hADSCs is age dependant. $\boldsymbol{A}$ and $\boldsymbol{B}$ show that hADSCs migrated robustly to the spleen and the liver area of young rats exposed to TBI relative to sham controls and to the spleen and liver area of aged rats exposed to TBI and transplanted with hADSCS (one-way ANOVA, $F_{(3,9)}=6.7, p<0.001$; Bonferroni's test, ${ }^{* * *} p<0.001$ ). C shows that the migration signal is significantly higher in aged rats only within the first $48 \mathrm{~h}$ compared with young and sham control (one-way ANOVA, $F_{(3,9)}=5.61, p<0.05$; Bonferroni's test, ${ }^{*} p<0.05$ ). The migration signal of the labeled hADSCs to the brain of young rats exposed to TBI was low and reached a plateau at $12 \mathrm{~h}$ after transplantation compared with aged rats and sham controls. D shows ex vivo near-infrared IVIS imaging of the brain, spleen, lungs, and liver $11 \mathrm{~d}$ after TBI. E, Ex vivo fluorescent analysis revealed that spleen and lungs of young rats exposed to TBI expressed higher migration of hADSCS compared with the spleen and lungs of rats exposed to TBI or sham controls; two-way ANOVA, $F_{(3,9)}=14.15, p<0.001$; Bonferroni's test, $\left.{ }^{*} p<0.05\right)$. Radiant Efficiency, photons per second per square centimeter per steridian divided by microwatts per square centimeter $\left(\frac{\mathrm{p} / \mathrm{s} / \mathrm{cm}^{2} / \mathrm{sr}}{\mu \mathrm{W} / \mathrm{cm}^{2}}\right)$. rats exposed to TBI and transplanted with hADSC-labeled cells expressed a higher fluorescent signal relative to the spleen and liver area of aged rats transplanted with hADSC-labeled cells (one-way ANOVA, $F_{(3,9)}=6.7, p<0.001$; Fig. $4 B$ ). By $24 \mathrm{~h}$ after transplantation, there were no significant differences in fluorescent signaling at the spleen and liver area of either young or aged rats $(p>0.05$; Fig. $4 B)$. The head region analyses revealed that only aged rats exposed to TBI showed a significantly higher signal within the first $24 \mathrm{~h}$ compared with young rats subjected to TBI. There was no fluorescent signal detected in the head region of sham controls (ANOVA, $F_{(3,9)}=5.61, p<0.05$; Fig. $4 C$ ). The signal of the labeled hADSCs to the brain of young rats exposed to TBI reached a plateau at $12 \mathrm{~h}$ after transplantation compared with aged rats and sham controls.

Age-dependent biodistribution and homing of systemically administrated hADSCs ex vivo

At day 11 after transplantation of hADSC, rats were killed, and brain, lungs, liver, and spleen were collected, imaged using the IVIS near-infrared spectrum imager, and processed for immunofluorescence (IF; Fig. $4 D, E$ ). Near-infrared IF showed that the biodistribution of hADSCs to organs displayed significant aged-related differences (two-way ANOVA, $F_{(3,9)}=$ 14.15, $p<0.001$; Fig. $4 E$ ). For the nearinfrared IVIS imaging, the spleen and lungs of young rats exposed to TBI showed higher fluorescent signals compared with the spleen and lungs of aged rats exposed to TBI or sham controls $(p<$ 0.05; Fig. $4 E$ ). Moreover, the florescence signal detected in the liver of aged rats exposed to TBI was higher relative to young rats subjected to TBI or sham controls $(p<0.05$; Fig. $4 E)$. There was no agerelated difference in fluorescence signals detected in the brains. To confirm that the fluorescence signal was related to surviving cells in the animals, we examined immunohistochemistry for HuNu. Less than $1 \%$ of the total cells injected were observed at this time after transplantation. Confocal photomicrographs of hADSC expression using IF demonstrated positive expression in brain and spleen from young and aged rats (Fig. 5). $\mathrm{HuNu}$ and Hoechst were used to detect the positive hADSCs in the brain and spleen. In the young rats, hADSCs appeared to survive in the brain, although as mentioned, $<1 \%$ of cells survive to this time after transplantation. Expression of $\mathrm{HuNu}$-positive cells 


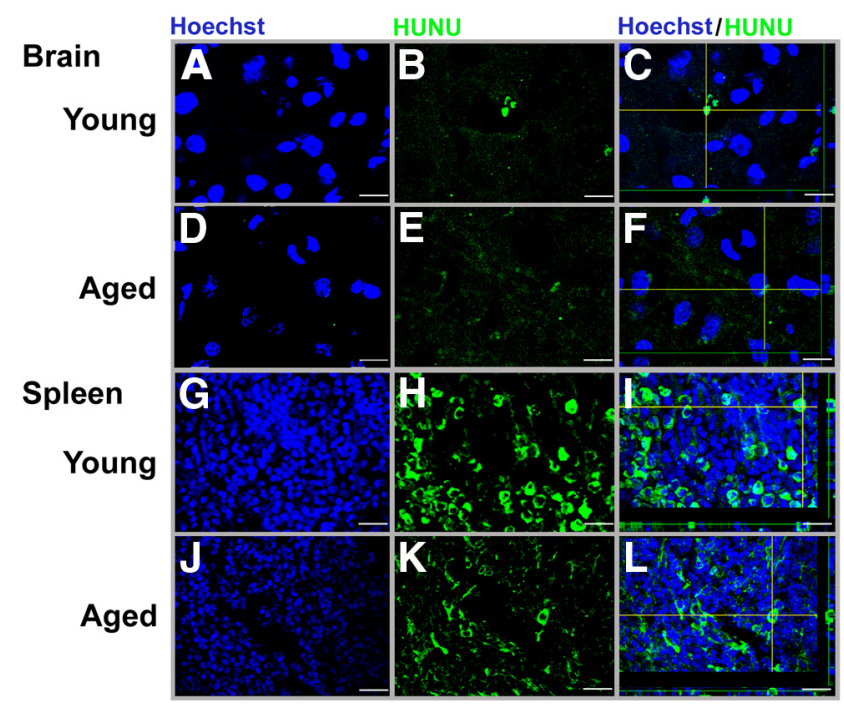

Figure 5. Confocal imaging of hADSC-positive expression in the brain and spleen of young and aged rats. Brain confocal images of positive HuNu (green) and Hoechst (blue) and expression of hADSCs (green) within the area of injury in the cortex of young $(\boldsymbol{A}-\boldsymbol{C})$ and aged $(\boldsymbol{D}-\boldsymbol{F})$ rats $11 \mathrm{~d}$ after transplantation. $\boldsymbol{C}$ and $\boldsymbol{F}$ show a confocal image of a $Z$-stack reconstruction of HuNu-positive cells colocalizing with Hoechst in young and aged rats, respectively. Spleen confocal images of HuNu- and Hoechst-positive expression of hADSCs in the spleen of young $(\mathbf{G}-\boldsymbol{I})$ and aged $(\boldsymbol{J}-\boldsymbol{L})$ rats $11 \mathrm{~d}$ after transplantation. $\boldsymbol{I}$ and $\boldsymbol{L}$ show confocal $Z$-stack reconstruction of hADSCs colocalizing with Hoechst in young and aged rats, respectively. I shows a robust migration of hADSCs in the spleen of young relative to the HuNu expression in the spleen of aged rats. Scale bars, $50 \mu \mathrm{m}$.

were found in the ipsilateral cortex within the area as demonstrated by the $Z$-stack reconstruction of $\mathrm{HuNu}$ and Hoechst colocalization. However, in the aged rat, there was a weak $\mathrm{HuNu}$ positive expression that can be associated with poor cell survival. Weak HuNu was found in the ipsilateral cortex within the area of injury. Analysis of the $Z$-stack reconstruction of the hADSC $(\mathrm{HuNu})$ expression in the spleen of young and aged rats demonstrated a higher migration and survival of hADSCs as depicted by their robust $\mathrm{HuNu} / \mathrm{Hoechst}$ expression in the young rats relative to aged rats. To further verify that the fluorescent signal observed in the spleen and other organs reflected hADSCs, we examined the spleens of three young rats after injury using flow cytometry. Although only a very small percentage of the cells in the spleen were DiR positive, $60 \%$ of the DiR-positive cells were CD105 positive, and none of the non-DiR cells were $\mathrm{CD} 105$ positive (data not shown). As discussed in Materials and Methods, the hADSCs used in this study are $80 \%$ CD105 positive at the time of injection; thus, a small percentage of DiR cells have either differentiated or DiR has migrated to other cells.

\section{hADSC transplantation reduces impact and peri-impact area of young rats exposed to TBI}

Nissl staining revealed that the administered hADSC graft treatment significantly reduced the impact and peri-impact area of TBI injury (one-way ANOVA, impact area, $F_{(3,28)}=10.54, p<$ 0.001 ; peri-impact, $F_{(3,28)}=22.98, p<0.001$; Fig. 6). Quantitative analyses revealed that there is a significant reduction of the damaged impacted area of the cortex of young rats treated with either hADSCs or CM relative to young rats treated with vehicle unconditioned media and sham control ( $p<0.05$; Fig. $6 B)$. Of note, there were no significant differences between young rats treated with hADSCs and CM ( $p>0.05$; Fig. $6 B)$. Moreover, Nissl analyses revealed a higher percentage of intact peri-impact area on the cortex of young rats exposed to TBI treated with hADSCs and CM relative to young rats treated with vehicle and sham controls $(p<0.05$; Fig. $6 C)$.

\section{hADSC transplantation partially ameliorates the cortical damage of aged rats subjected to TBI}

Nissl staining revealed that treatment of hADSCs or CM was not able to reduce the damage of the impacted area of the cortex in aged rats compared with aged rats treated with vehicle media and sham controls $(p>0.05$; Fig. $6 E)$. However, Nissl staining showed that hADSC treatment partially decreased the damage of the cortex in the peri-impact area (ANOVA, $F_{(3,28)}=30.60, p<$ 0.001 ; Fig. $6 F$ ). Quantitative analyses revealed that the percentage of intact peri-impact area of aged rats treated with hADSCs does not differ from aged sham control, and it is significantly greater relative to aged $\mathrm{CM}$ and aged vehicle-treated rats $(p<0.0001$; Fig. $6 F)$.

\section{hADSC grafts and CM decrease hippocampal cell loss in} young rats exposed to TBI but not in the aged rats

The total number of surviving neurons in the hippocampal CA3 region of young and aged rats exposed to TBI was quantified by H\&E staining (Fig. 7). Treatment with hADSCs or CM significantly increases neuronal cells survival in the $\mathrm{CA} 3$ region of the hippocampus (one-way ANOVA, $F_{(3,28)}=7.8, p<0.001$; Fig. 7). There was a significant decrease on the percentage of neuronal cell loss in the young rats exposed to TBI and treated with either hADSCs or CM compared with young vehicle unconditioned media and sham controls $(p<0.05$; Fig. 7A). Quantitative analyses failed to reveal a significant reduction in CA3 neuronal cell loss in aged rats that received hADSCs or CM treatment grafts $(p>0.05$; Fig. $7 B)$.

Knockdown of NEAT1 and MALAT1 blocks the CM-mediated motor behavioral improvements in young rats exposed to TBI To decipher the therapeutic mechanism underlying the observed therapeutic benefits of hADSCs, an additional cohort of young rats was used to test two specific lncRNAs found to be secreted by hADSCs into media. Because knockdown of transcription factors can also alter other biomolecules secreted by the hADSCs into the $\mathrm{CM}$, we also examined three potential bioactive molecules observed in CM. When CM was compared with unconditioned media, we identified 13 proteins that were increased in CM compared with unconditioned media (Table 1); of these, we chose to examine three proteins that have been suggested to have effects on stem cell survival: stem cell factor (SCF), vascular endothelial growth factor (VEGF), and tissue inhibitor of metalloproteinases-3 (TIMP3). As observed in Table 2, knockdown treatment of hADSCs with antisense to NEAT1 or MALAT1 reduces the amount of NEAT1 or MALAT1 in the CM. In addition, there is also a significant reduction in VEGF and SCF measured in the CM (VEGF, one-way ANOVA, $F_{(3,19)}=482, p<$ 0.001; post hoc tests revealed that CM was different from either $\operatorname{kdNEAT} 1$ or $\operatorname{kdMALAT} 1$ at $p<0.001$; SCF, $F_{(3,19)}=336.4, p<$ 0.001; post hoc tests revealed the difference between CM and kdNEAT1 or kdMALAT1 at $p<0.001)$. When we examined TIMP3 in the $\mathrm{CM}$, there was an increase in the CM from the cells treated with antisense to NEAT1 or MALAT1 compared with CM (oneway ANOVA, $F_{(3,17)}=59.51, p<0.0001 ;$ post hoc tests, $p<0.001$ comparing CM with kdNEAT1 or kdMALAT1).

We observed that either CM kdNEAT1 or CM kdMALAT1 reduced the therapeutic effects seem previously with the treatment of CM when compared with sham control (two-way 
A

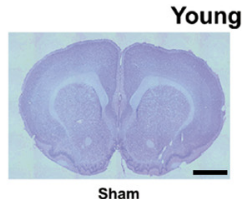

Sham

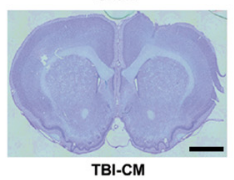

B

$\%$ Impact Area Young rats

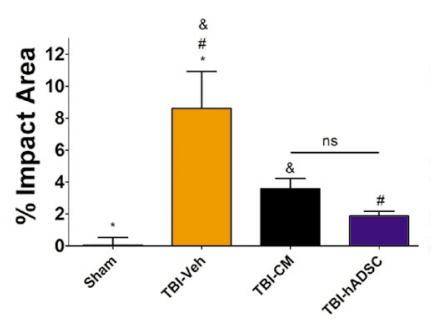

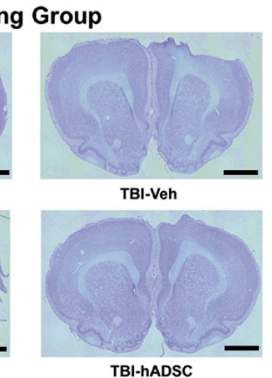

D

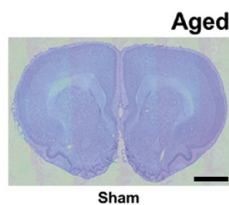

Sham
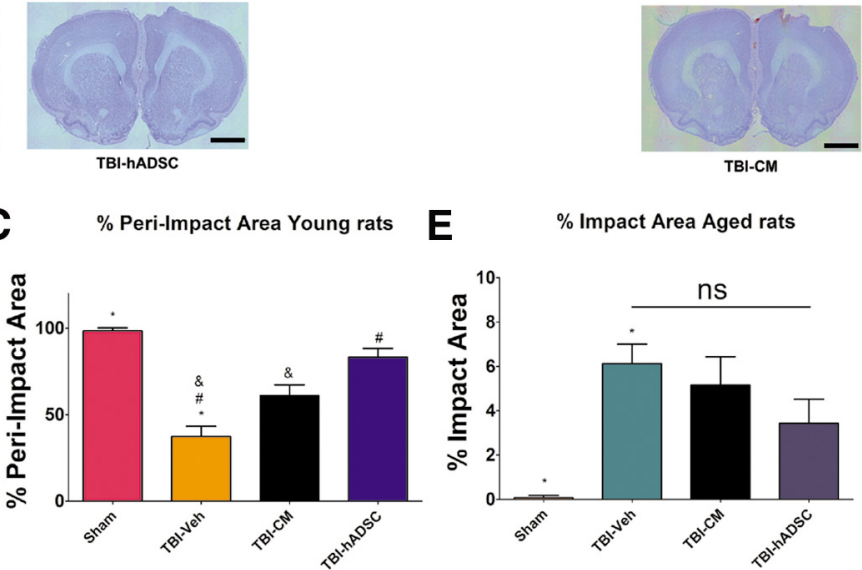

$\%$ Impact Area Aged rats

ns

E

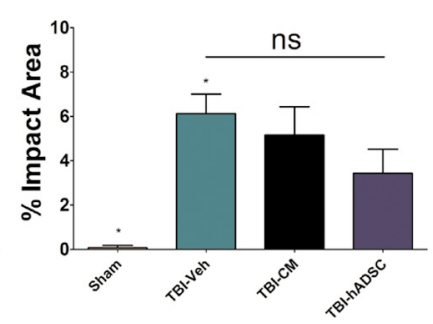

Aged Group

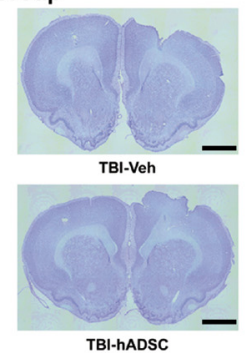

F

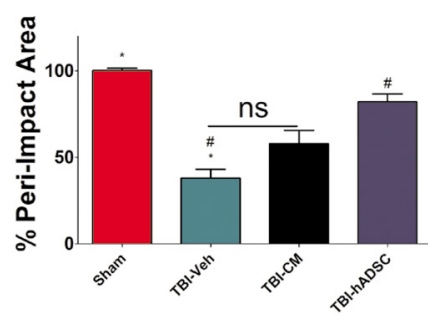

Figure 6. hADSC transplantation reduces impact and peri-impact area of young rats exposed to TBI. Nissl staining revealed that hADSC treatment significantly reduced the cortical damage at the impact and peri-impact area associated with TBI injury in young rats. $A$, Photomicrographs of brain coronal sections of sham-, vehicle-, CM-, or hADSC-treated young rats. $\boldsymbol{B}$, There is a 50 and $80 \%$ reduction of impacted area after treatment with CM and hADSCS, respectively. C, Graphs show that the intact peri-impact area increased 40 and $75 \%$ after treatment of CM and hADSCS, respectively (one-way ANOVA, impact area, $F_{(3,28)}=10.54, p<0.001$; peri-impact, $F_{(3,28)}=22.98, p<0.001 ; A-C$ ). Impact and peri-impact analysis of aged rats revealed that treatment of hADSCs partially rescued the TBI-associated cortical damage only in the peri-impact area compared with vehicle and sham controls (D-F). $\boldsymbol{D}$, Photomicrographs of brain coronal sections of sham-, vehicle-, $(\mathrm{CM}-$, or hADSC-treated aged rats. $\boldsymbol{E}$, Graph shows no significant differences among different treatment in the impact area of aged rats. $\boldsymbol{F}$, Graph shows that only hADSC treatment increased intact peri-impact area after TBI (ANOVA, peri-impact area, $F_{(3,28)}=30.60, p<0.0001$ ). Scale bars, $1 \mathrm{~mm}$. ${ }^{*}$, Significantly different from sham control; \&, significantly different from TBI-CM; \#, significantly different from TBI-hADSC; ns, not significant using Bonferroni's test.

ANOVA, EBST, $F_{(3,26)}=61.34, p<0.001$; forelimb akinesia, $F_{(3,26)}=58.92, p<0.001$; paw grasp, $F_{(3,26)}=36.92, p<0.001$; Fig. 8). EBST demonstrated significant asymmetry in motor activity at days 1,3 , and 7 after TBI $(p<0.01)$ in rats treated with either kdNEAT1 or kdMALAT1 relative to CM, CM kdscramble, and sham control (Fig. 8A). Moreover, blocking either NEAT1 or MALAT1 lowered the efficacy of the CM to improve motor functional performance on forelimb akinesia and the paw-grasp test $(p<0.05$; Fig. $8 B, C)$. These observations suggest that lncRNAs, NEAT1, and MALAT1 play a key role in sustaining long-term neuroprotection and behavioral improvement.

\section{Knockdown of NEAT1 and MALAT1 blocks the hADSC- and CM-mediated amelioration of cognitive impairments in young TBI rats}

Young sham controls subjected to TBI treated systemically with CM kdNEAT1, CM kdMALAT1, CM kdscramble, or CM were tested for spatial memory using the six-arm spaced training RAWM task (Fig. 9). As described previously in this study, the rats were trained for four trials a day for 3 consecutive days to find the hidden escape platform. During the acquisition of learning, there were no differences in performance, demonstrating that all groups of rats, young and aged, were able to learn the location of the hidden platform ( $p>0.05$; Fig. 9A). Of note, there were no disparities of motor function and swimming capabilities, indicating that they equally escaped from the water by finding the platform during the allotted $60 \mathrm{~s}$ learning trials. Administration of CM kdNEAT1 or CM kdMALAT1 significantly abrogated the therapeutic benefits seen previously because hADSCs or CM treatment ameliorated the TBI-related memory impairments in the spatial navigation task (one-way ANOVA, $F_{(3,26)}=4.129, p<$ 0.01 ; Fig. $9 B$ ). All data are represented as mean \pm SEM values. There was no amelioration of cognitive function in young rats treated with CM kdNEAT1 and CM kdMALAT1 relative to CM, CM kdscramble, and sham control $(p<0.05)$.

\section{Knockdown of NEAT1 and MALAT1 blocks the hADSC- and $\mathrm{CM}$-mediated reduction in hippocampal cell loss in young rats exposed to TBI}

After systemic administration of kd lncRNAs and appropriate controls, the total number of surviving neurons in the hippocampal CA3 region of young rats was quantified by H\&E staining (Fig. 10). Administration of CM kdNEAT1 and CM kdMALAT1 significantly blocked the therapeutic effects relative to $\mathrm{CM}, \mathrm{CM}$ kdscramble, and sham control (one-way ANOVA, $F_{(5,31)}=$ 23.24, $p<0.001$; Fig. 10). There was no therapeutic effect to reduce the TBI-associated neuronal cell loss in young rats treated with CM kdNEAT1 and CM kdMALAT1 compared with CM, CM kdscramble, and sham control ( $p>0.05$; Fig. 10).

\section{Discussion}

This study reports the therapeutic potential of hADSC or CM from hADSC in young and aged rats after TBI, characterized by attenuation of TBI-induced motor, cognitive, and histological deficits, possibly via endogenous repair mechanisms promoted by lncRNA. We demonstrate that hADSC transplantation or CM attenuated the TBI-associated motor and cognitive impairments in young rats but had decreased efficacy in the aged rats.

\section{hADSCs and CM afford neuroprotective effects}

In the present in vivo study, behavioral and histological ameliorations were achieved by hADSC transplantation and CM treatment. The decreased cortical damage and CA3 neuronal loss may directly relate to the motor and cognitive improvement seen in young rats after hADSC treatment. In contrast, hADSC treatment in aged rats only partially rescued the cortical damage, and 
A \% CA3 Neuronal Loss Young rats

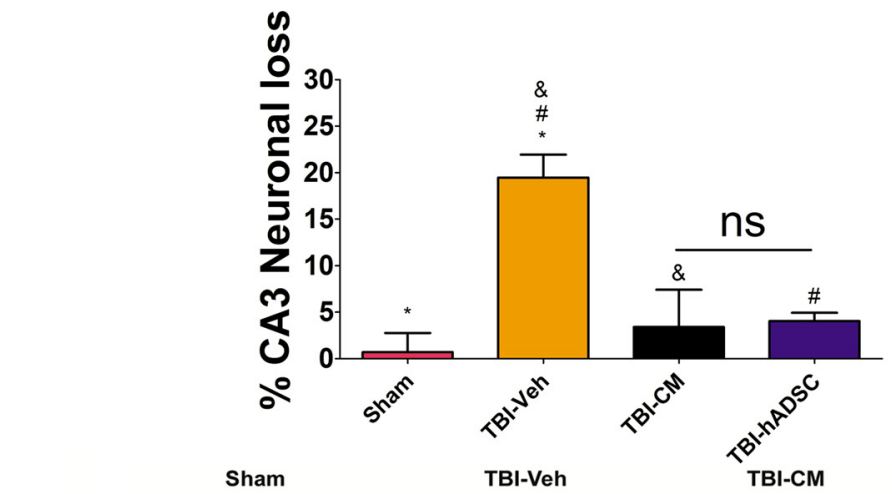

\section{B \% CA3 Neuronal Loss Aged rats}

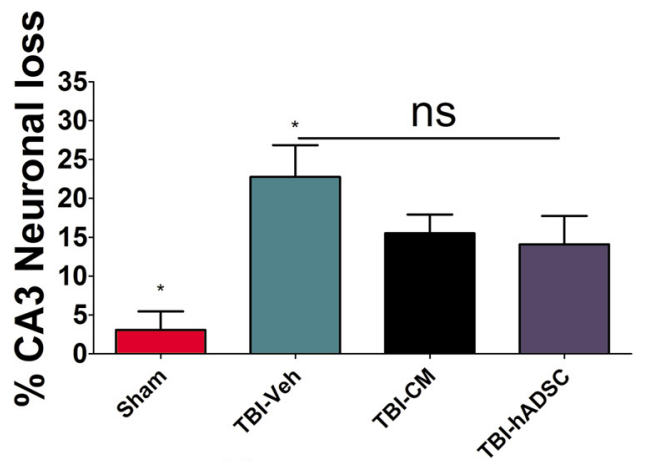

TBI-hADSC

K

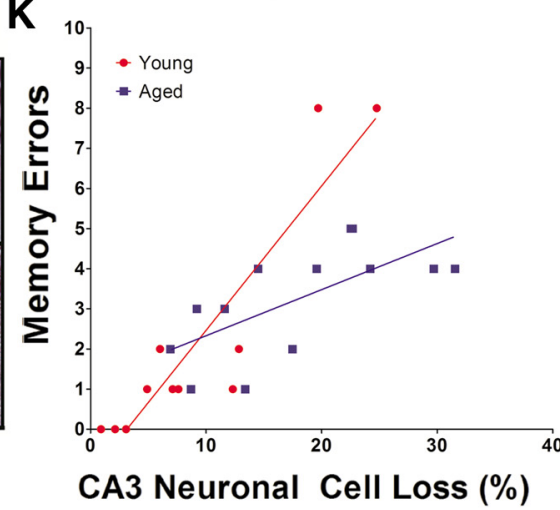

Figure 7. hADSC treatment reduces hippocampal cell loss in young TBI rats. Results show that hADSC or CM treatments are neuroprotective in the hippocampus of young rats exposed to TBI and not in aged rats. $\boldsymbol{A}$ shows that there is a significant reduction of the percentage of CA3 neuronal cell loss after treatment with CM or hADSCs compared with vehicle-treated young TBI rats or sham controls (one-way ANOVA, $F_{(3,28)}=7.8, p<0.001$ ). B, Quantitative analyses failed to reveal a significant reduction on the percentage of CA3 neuronal cell loss of aged TBI rats after treatment with hADSC grafts relative to aged vehicle and aged sham control rats $(p>0.05)$. C $-J$, Photomicrographs of H\&E staining of ipsilateral dorsal hippocampus, specifically the CA3 region of the hippocampus, in sham, TBI-vehicle, TBI-CM, and TBI-hADSC. Arrows denote neuronal cell loss within the CA3 region. $K$ illustrates the correlation between the number of errors made on trial 4 of the reversal trial (taken from Fig. 3 ) with the loss of CA3 neuronal cells. There is a significant correlations between memory and CA3 neuronal loss when examined within age groups and between age groups $\left(R^{2}\right.$ for young $=0.82$; aged $=0.37$; combined $=0.60$; Pearson's $r$ for young $=0.91$; aged $=0.61$; combined 0.78$)$. Scale bars, $50 \mu \mathrm{m}$. ${ }^{*}$, Significantly different from sham control; $\&$, significantly different from TBI-CM; \#, significantly different from TBI- hADSC; ns, not significant, using Bonferroni's test.

Table 1. Major proteins secreted into conditioned media

\begin{tabular}{lcccc}
\hline Factor & Units & UCM & CM & Fold change CM/UCM \\
\hline Adiponectin & $\mathrm{ng} / \mathrm{ml}$ & $<0.29$ & 0.58 & $>2$ \\
B2M & $\mu \mathrm{g} / \mathrm{ml}$ & 0.0011 & 0.011 & 10 \\
Compliment C3 & $\mathrm{ng} / \mathrm{ml}$ & 0.2 & 2.4 & 12 \\
IL-6 & $\mathrm{pg} / \mathrm{ml}$ & $<0.78$ & 400 & $>100$ \\
IL-8 & $\mathrm{pg} / \mathrm{ml}$ & 1.2 & 12 & 10 \\
MMP-3 & $\mathrm{ng} / \mathrm{ml}$ & $<0.046$ & 0.14 & $>3$ \\
MCP-1 & $\mathrm{pg} / \mathrm{ml}$ & $<9.1$ & 191 & $>20$ \\
PAl-1 & $\mathrm{ng} / \mathrm{ml}$ & $<0.031$ & 259 & $>100$ \\
SCF & $\mathrm{pg} / \mathrm{ml}$ & $<12$ & 13 & $>1$ \\
TIMP-1 & $\mathrm{ng} / \mathrm{ml}$ & 0.05 & 45 & 900 \\
TNFR2 & $\mathrm{ng} / \mathrm{ml}$ & 0.006 & 0.013 & 2.2 \\
VCAM-1 & $\mathrm{ng} / \mathrm{ml}$ & $<0.025$ & 0.067 & $>2$ \\
VEGF & $\mathrm{pg} / \mathrm{ml}$ & 15 & 247 & 16.5 \\
\hline
\end{tabular}

MCP-1, Monocyte chemoattractant protein; MMP-3, matrix metalloproteinase-3; PAI-1, plasminogen activator inhibitor-1; TNFR2, TNF receptor-2; UCM, unconditioned medium; VCAM-1, vascular cell adhesion molecule 1.

it has no significant neuroprotective effect in the CA3 region of the hippocampus. Consequently, as seen in Results, there was no improvement of the motor deficits and only partial amelioration on the cognitive function in the aged rats. Previously, hADSCs have been shown to enhance synaptic plasticity with subsequent reconstruction of the cortical neuronal network (Mizuno et al., 2012). Certainly, the underlying mechanisms might include functional enhancement of the surviving neurons by either cell- to-cell interaction or the bystander effect of the CM from the stem cells, which contain a variety of secreted factors, such as chemokines, cytokines, trophic factor, and lncRNA that serve as signaling molecules and anti-inflammatory mediators and might intervene in epigenetic mechanisms for neuroprotection and synaptic plasticity (Egashira et al., 2012; Ribeiro et al., 2012).

\section{Biodistribution of hADSCs}

It is now well established that, when cell therapy is administered peripherally as was done in this investigation, the cells migrate to many organs. Migration of cells to the spleen has been implicated as part of the neuroprotective action, as has been shown after both stroke and TBI; the spleen shrinks in the first few days, but this is prevented with stem cell therapy (Vendrame et al., 2006; Walker et al., 2010). Walker et al. (2010) have shown that, after TBI, multipotent adult progenitor cells migrate to the spleen and prevent the shrinkage normally observed after injury. Early studies from our group demonstrated that intravenous cell therapy has important influences on the monocytes and macrophages in the spleen as an important neuroprotective action in stroke (Vendrame et al., 2006). Additional studies demonstrated that removal of the spleen immediately after TBI (Li et al., 2011) or before stroke (Ajmo et al., 2008) significantly reduced proinflammatory cytokines and improved cognitive function. For this reason, we examined the migration of hADSCs after treatment. 
Table 2. Effect of knockdown of NEAT1 or MALAT1 on conditioned media

\begin{tabular}{|c|c|c|c|c|c|}
\hline Media condition & NEAT1 (\% control) & MALAT1 (\% control) & VEGF (\% control) & SCF (\% control) & TIMP3 (\% control) \\
\hline Control CM & $100 \pm 3$ & $100 \pm 5$ & $100 \pm 8$ & $100 \pm 8$ & $100 \pm 7$ \\
\hline Antisense NEAT1 & $15.3 \pm 5^{\#}$ & $71.5 \pm 5$ & $27.6 \pm 15^{\#}$ & $28.4 \pm 3^{\#}$ & $400 \pm 26^{\#}$ \\
\hline Antisense MALAT1 & $87.4 \pm 4$ & $5.5 \pm 4^{\#}$ & $42.8 \pm 24^{\#}$ & $63.1 \pm 8^{\#}$ & $290 \pm 32^{\#}$ \\
\hline
\end{tabular}

\section{A EBST TBI CM-Kd IncRNA}

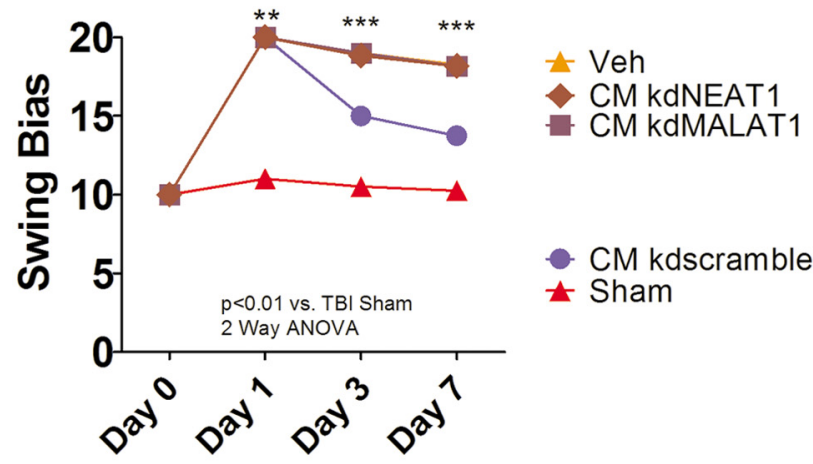

\section{B Forelimb Akinesia CM-Kd IncRNA}

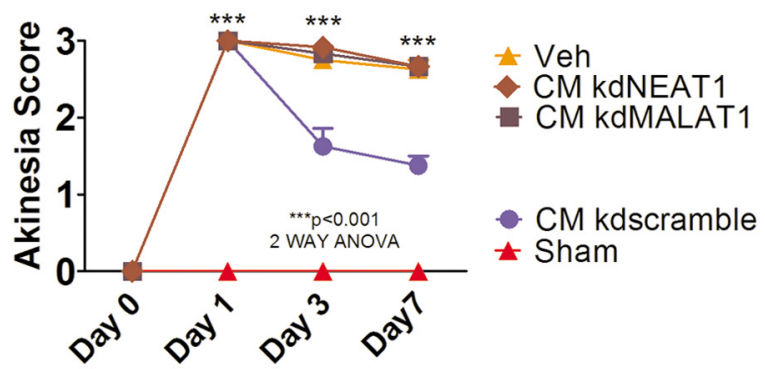

\section{Paw Grasp CM-Kd IncRNA}

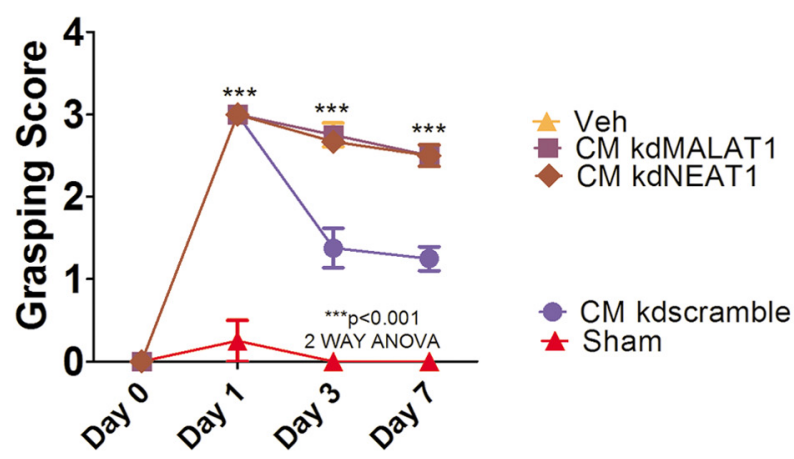

Figure 8. Knockdowns of NEAT1 and MALAT1 block the CM-mediated motor behavioral improvements in young rats exposed to TBI. Results indicate that treatment of CM kdNEAT1 or CM kdMALAT1 reduced the therapeutic effects seen previously with the treatment of $C M$ when compared with sham control (two-way ANOVA, EBST, $F_{(3,26)}=61.34, p<0.0001$; Bonferroni's test, ${ }^{* *} p<$ 0.01 , ${ }^{* * *} p<0.001$; forelimb akinesia, $F_{(3,26)}=58.92, p<0.001$; Bonferroni's test, ${ }^{* * *} p<0.001$; paw grasp, $F_{(3,26)}=36.92, p<0.0001$; Bonferroni'stest, $\left.{ }^{* * *} p<0.001\right) . A$, EBST revealed significant asymmetry in days 1,3 , and 7 after TBI in rats treated with either kdNEAT1 or kdMALAT1 relative to CM, CM kdscramble, and sham control. $\boldsymbol{B}$ and $\boldsymbol{C}$ also demonstrate a decrease in forelimb akinesia and paw grasp improvements in days 1, 3, and 7 by blocking either NEAT1 or MALAT1.

In recent years, several techniques have been applied to track stem cell migration and survival in vivo, including MRI and optical imaging (Solanto, 1984; Ushiki et al., 2010; Darkazalli and Levenson, 2012). By using DiR dye, we can easily trace cells in vivo, even through the skull because of its low level of autofluorescence combined with a high wavelength (Michalet et al., 2005;
Boddington et al., 2008; Jang et al., 2011; Sugiyama et al., 2011). By using in vivo optical imaging, the present study clearly demonstrates that a small percentage of hADSCs survive and are observed in the injured brain as early as $12 \mathrm{~h}$ after injury. The majority of DiR-labeled cells were detected in the spleen/liver region within $1 \mathrm{~h}$ after transplantation. A similar pattern of in vivo biodistribution was reported (Detante et al., 2009) after middle cerebral artery occlusion using $99 \mathrm{mTc}$-hMSC cells into the saphenous vein. An important observation of this study was that the biodistribution of cells differed in the aged rats after TBI with a higher signal in the aging head, whereas there was a lower signal in the spleen/liver region of aged rats in the subacute stage after TBI and confirmed lower signal in the spleen of aged rats with the ex vivo imaging. The signal observed in the brain was significantly higher at $24 \mathrm{~h}$. One explanation for this is that there is a larger disruption of the blood-brain barrier in the aged versus young rats (Lee et al., 2012). Despite increased signal at early time points to the aging brain within the subacute stage, these cells failed to fully rescue TBI-mediated motor and cognitive impairment. There are several potential reasons for the reduced efficacy of the cell therapy in the aged rats. First, as noted, there were fewer $\mathrm{HuNu}$-positive cells observed in the brains of the aged rats, and those $\mathrm{HuNu}$ cells that are still present appeared to be dying. This was not observed in the young brain. Thus, one part of the observed difference in efficacy of cell therapy could be related to cell survival in the aged brain and/or spleen. Second, it is possible that the damage is more severe in the aged brain and thus less responsive to therapy. Third, the reduced migration to the spleen may be a critical aspect of the reduced efficacy of the hADSCs in the aged rats. As discussed above, this is thought to be one important aspect of the mechanism of action of cell therapy after brain injury (Walker et al., 2010). The exact reason why hADSCs might show decreased migration to the spleen in the aged rats is not currently established. One explanation could be the aged-related decrease in the chemokine profile in aged rats. hADSCs constitutively express chemokine receptors CCR7, CXCR5, and CXCR6 for their corresponding homeostatic chemokines CCL19, CCL21, and CCL20, respectively, which play an important role in cell migration and homing (Baek et al., 2011). Recent studies showed a significantly lower amount of the chemokines CCL19, CCL21, and CCL20 in aged mouse spleen compared with young mice (McDonald et al., 2011; Lefebvre et al., 2012). Thus, additional studies need to examine whether approaches aimed at increasing these chemokines in the aged spleen could modulate the efficacy of stem cell therapy in the aged. Other approaches could be directed at mechanisms to increase cell survival in the aged animals, because a possible caveat for fewer cells observed in the aged spleen at $11 \mathrm{~d}$ after injection is that fewer cells are surviving in the aged spleen, as we suggested may be happening in the aged brain.

\section{Mechanism of CM efficacy after TBI}

One mechanism suggested to play a role in the therapeutic action of hADSCs is that they secrete many important factors. An analysis of the CM used in the experiments described here shows an 


\section{A Young rats /Knockdown NEAT1 and MALAT1 B Young rats/Knockdown NEAT1 and MALAT1}
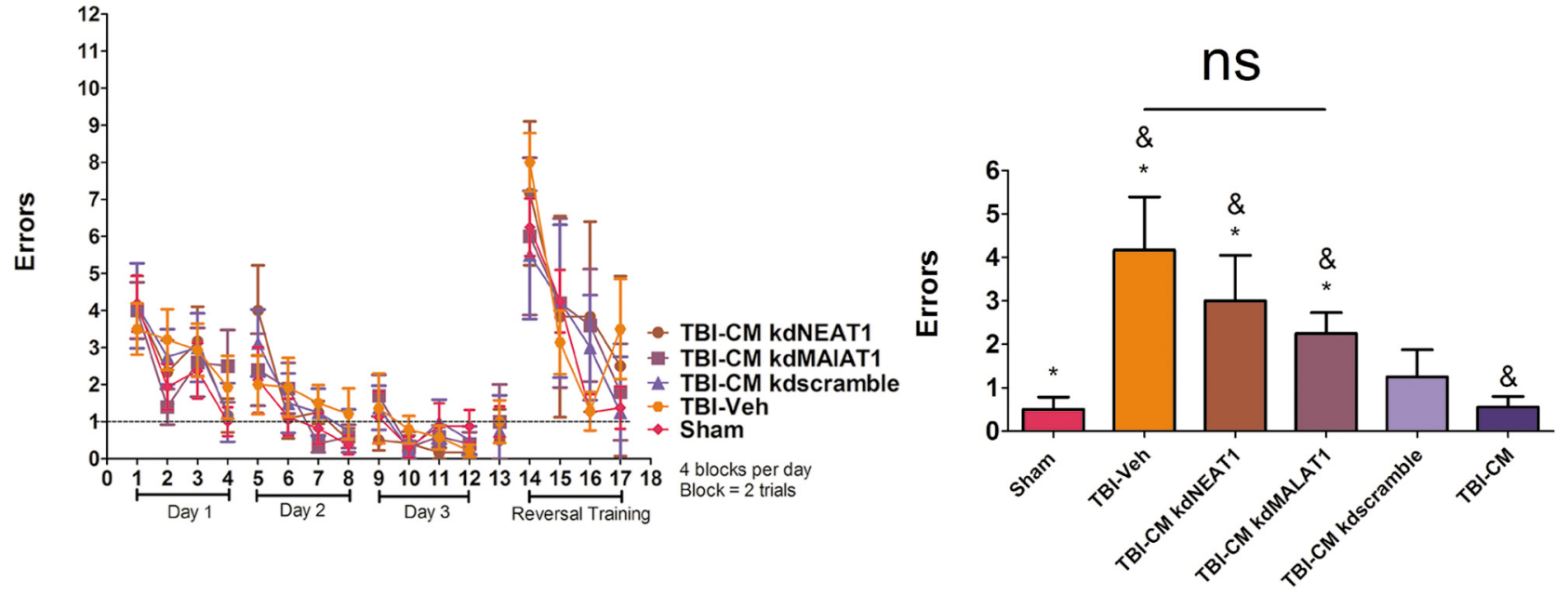

Figure 9. Knockdown of NEAT1 and MALAT1 blocks the hADSC- and CM-mediated amelioration of cognitive impairments in young TBI rats. Results demonstrated that administration of CM kdNEAT1 or CM kdMALAT1 significantly abrogated the therapeutic benefits seen previously as hADSCs or CM treatment ameliorated the TBI-related memory impairments in the spatial navigation task (one-way ANOVA, $\left.F_{(3,26)}=4.129, p<0.01\right)$. There was no amelioration of cognitive function in young rats treated with CM kdNEAT1 and CM kdMALAT1 relative to CM, CM kdscramble, and sham control $(p>0.05)$. A shows escape errors (mean \pm SEM) to find the hidden platform in the RAWM for days $1-3$ (4 blocks per day; a block is 2 trials) and for reversal testing on day 4 (trials $1-4$ ) for young rats. $\boldsymbol{B}$ shows trial 4 from the reversal training. ${ }^{*}$, Significantly different from sham control; \&, significantly different from TBI-CM; ns, not significant using Bonferroni's test.

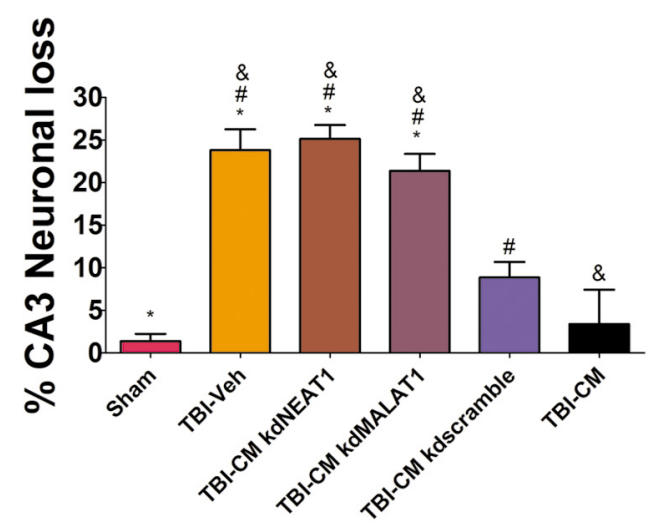

Figure 10. Knockdown of NEAT1 and MALAT1 blocks the hADSC- and CM-mediated reduction in hippocampal cell loss in young rats exposed to TBI. Administration of CM kdNEAT1 and CM kdMALAT1 significantly blocked the therapeutic effects relative to $C M, C M$ kdscramble, and sham control (one-way ANOVA, $F_{(5,31)}=23.24, p<0.001$ ). Graph shows the percentage loss of CA3 hippocampal neurons. There is no therapeutic effects to reduce the TBI-associated neuronal cell loss in young rats treated with CM kdNEAT1 or CM kdMALAT1 compared with vehicle, CM, CM kdscramble, or sham control ( $p>0.05)$. ${ }^{*}$, Significantly different from sham control; \&, significantly different from TBI-CM; \#, significantly different from TBI-CM kdscramble using Bonferroni's test.

increase in NEAT and MALAT and a number of important proteins, such as VEGF and TIMP. It has been shown that hADSC $\mathrm{CM}$ enhances neuronal and glial viability and is neuroprotective in stroke (Cho et al., 2012; Egashira et al., 2012). It has been shown that hADSCs produce massive secretion of antiinflammatory cytokines IL-10 and IL-4 in experimental TBI (Galindo et al., 2011; Egashira et al., 2012; Ribeiro et al., 2012) and decrease production of proinflammatory cytokines, such as TNF- $\alpha$ and IFN- $\gamma$ (Blaber et al., 2012), when cocultured with immune cells, effector T cells, natural killer NK cells, and dendritic cells (Singer and Caplan, 2011). Because CM showed similar therapeutic potential in young animals, next we examined lncRNAs, which have been postulated previously as regulators of epigenetic mechanisms. hADSC-secreted exosomes carry a com- plex cargo load of lncRNA (Katsuda et al., 2013), which has been shown previously to improve cardiac function by reducing oxidative stress and inducing the phosphatidylinositol 3-kinase/Akt signaling pathway (Arslan et al., 2013). There are no published reports on the involvement of lncRNAs in rescuing neurons after brain injury, although over the past decade, there is emerging evidence that lncRNA serves as a key regulator in cell proliferation and survival in mammalian cells (Spadaro and Bredy, 2012; Mercer and Mattick, 2013). When expression of NEAT1 or MALAT1 in CM is knocked down with their corresponding antisense RNA, CM loses its efficacy to provide neuroprotection and behavioral improvements. It has been shown that NEAT1 and MALAT1 are essential components of nuclear speckles and chromatin (Bond and Fox, 2009; Clemson et al., 2009; Sasaki et al., 2009; Naganuma and Hirose, 2013), which control stress responses and cellular differentiation (Nakagawa and Hirose, 2012). In addition, MALAT1 significantly influences synapse formation in neuronal culture by controlling the expression of a subset of genes significantly involved in nuclear and synapse function, such as neuroligin 1 and synaptic cell adhesion molecule 1 (Bernard et al., 2010). We postulated that, after TBI, reservoir paraspeckles are released into the cytoplasm (Fox and Lamond, 2010), and NEAT1 and MALAT1 play a major role in distributing the pre-mRNA splicing factors to nuclear speckles and thus maintain the regulatory network of brain plasticity. In addition to the reduction in NEAT1 or MALAT1, we also examined three biomolecules with potential importance for brain repair. Treatment of hADSCs with antisense to either NEAT1 or MALAT1 partially reduced the presence of both VEGF and SCF and increased the expression of TIMP3, which is an inhibitor of VEGF (Qi et al., 2003; Yao et al., 2012). All three of these proteins have been shown to have biological activity in repair after brain injury and thus may also be involved in the protective effect of CM observed in this study. Future studies can address the relative importance of NEAT or MALAT individually.

In conclusion, our results suggest that hADSC cell transplantation and CM from hADSCs have the potential to boost endog- 
enous repair mechanisms as observed in the ability to improve motor and cognitive behaviors, as well as prevention of cortical and hippocampal damage. We demonstrate two major findings. First, lncRNA found in exosomes secreted by hADSCs plays a major role in the efficacy of the CM and, by extrapolation, plays a role in the actions of the transplanted hADSCs. Second, in aged rats, there is a decreased efficacy of the hADSCs and CM, and we propose that this is related to the reduced migration of hADSCs and/or reduced survival of hADSCs in the aged and potentially reduced migration of exosomes carrying lncRNA to the spleen. These findings directly advance our basic scientific knowledge about a potent mechanism of brain repair in TBI and provide pivotal guidance into the translational applications of cell therapy to TBI patients.

\section{References}

Acosta SA, Tajiri N, Shinozuka K, Ishikawa H, Grimmig B, Diamond D, Sanberg PR, Bickford PC, Kaneko Y, Borlongan CV (2013) Long-term upregulation of inflammation and suppression of cell proliferation in the brain of adult rats exposed to traumatic brain injury using the controlled cortical impact model. PLoS One 8:e53376. CrossRef Medline

Ajmo CT Jr, Vernon DO, Collier L, Hall AA, Garbuzova-Davis S, Willing A, Pennypacker KR (2008) The spleen contributes to stroke-induced neurodegeneration. J Neurosci Res 86:2227-2234. CrossRef Medline

Arslan F, Lai RC, Smeets MB, Akeroyd L, Choo A, Aguor EN, Timmers L, van Rijen HV, Doevendans PA, Pasterkamp G, Lim SK, de Kleijn DP (2013) Mesenchymal stem cell-derived exosomes increase ATP levels, decrease oxidative stress and activate PI3K/Akt pathway to enhance myocardial viability and prevent adverse remodeling after myocardial ischemia/reperfusion injury. Stem Cell Res 10:301-312. CrossRef Medline

Baek SJ, Kang SK, Ra JC (2011) In vitro migration capacity of human adipose tissue-derived mesenchymal stem cells reflects their expression of receptors for chemokines and growth factors. Exp Mol Med 43:596-603. CrossRef Medline

Bernard D, Prasanth KV, Tripathi V, Colasse S, Nakamura T, Xuan Z, Zhang MQ, Sedel F, Jourdren L, Coulpier F, Triller A, Spector DL, Bessis A (2010) A long nuclear-retained non-coding RNA regulates synaptogenesis by modulating gene expression. EMBO J 29:3082-3093. CrossRef Medline

Bjorklund LM, Sánchez-Pernaute R, Chung S, Andersson T, Chen IY, McNaught KS, Brownell AL, Jenkins BG, Wahlestedt C, Kim KS, Isacson O (2002) Embryonic stem cells develop into functional dopaminergic neurons after transplantation in a Parkinson rat model. Proc Natl Acad Sci U S A 99:2344-2349. CrossRef Medline

Bjugstad KB, Teng YD, Redmond DE Jr, Elsworth JD, Roth RH, Cornelius SK, Snyder EY, Sladek JR Jr (2008) Human neural stem cells migrate along the nigrostriatal pathway in a primate model of Parkinson's disease. Exp Neurol 211:362-369. CrossRef Medline

Blaber SP, Webster RA, Hill CJ, Breen EJ, Kuah D, Vesey G, Herbert BR (2012) Analysis of in vitro secretion profiles from adipose-derived cell populations. J Transl Med 10:172. CrossRef Medline

Boddington S, Henning TD, Sutton EJ, Daldrup-Link HE (2008) Labeling stem cells with fluorescent dyes for non-invasive detection with optical imaging. J Vis Exp (14):pii:686. CrossRef

Bond CS, Fox AH (2009) Paraspeckles: nuclear bodies built on long noncoding RNA. J Cell Biol 186:637-644. CrossRef Medline

Borlongan CV, Sanberg PR (1995) Elevated body swing test: a new behavioral parameter for rats with 6-hydroxydopamine-induced hemiparkinsonism. J Neurosci 15:5372-5378. Medline

Borlongan CV, Hida H, Nishino H (1998) Early assessment of motor dysfunctions aids in successful occlusion of the middle cerebral artery. Neuroreport 9:3615-3621. CrossRef Medline

Carlson ME, Conboy IM (2007) Loss of stem cell regenerative capacity within aged niches. Aging Cell 6:371-382. CrossRef Medline

Cho YJ, Song HS, Bhang S, Lee S, Kang BG, Lee JC, An J, Cha CI, Nam DH, Kim BS, Joo KM (2012) Therapeutic effects of human adipose stem cellconditioned medium on stroke. J Neurosci Res 90:1794-1802. CrossRef Medline

Clarkson ED (2001) Fetal tissue transplantation for patients with Parkin- son's disease: a database of published clinical results. Drugs Aging 18:773785. CrossRef Medline

Clemson CM, Hutchinson JN, Sara SA, Ensminger AW, Fox AH, Chess A, Lawrence JB (2009) An architectural role for a nuclear noncoding RNA: NEAT1 RNA is essential for the structure of paraspeckles. Mol Cell 33: 717-726. CrossRef Medline

Conboy IM, Conboy MJ, Wagers AJ, Girma ER, Weissman IL, Rando TA (2005) Rejuvenation of aged progenitor cells by exposure to a young systemic environment. Nature 433:760-764. CrossRef Medline

Darkazalli A, Levenson CW (2012) Tracking stem cell migration and survival in brain injury: current approaches and future prospects. Histol Histopathol 27:1255-1261. Medline

Derrien T, Johnson R, Bussotti G, Tanzer A, Djebali S, Tilgner H, Guernec G, Martin D, Merkel A, Knowles DG, Lagarde J, Veeravalli L, Ruan X, Ruan Y, Lassmann T, Carninci P, Brown JB, Lipovich L, Gonzalez JM, Thomas M, Davis CA, Shiekhattar R, Gingeras TR, Hubbard TJ, Notredame C, Harrow J, Guigó R (2012) The GENCODE v7 catalog of human long noncoding RNAs: analysis of their gene structure, evolution, and expression. Genome Res 22:1775-1789. CrossRef Medline

Detante O, Moisan A, Dimastromatteo J, Richard MJ, Riou L, Grillon E, Barbier E, Desruet MD, De Fraipont F, Segebarth C, Jaillard A, Hommel M, Ghezzi C, Remy C (2009) Intravenous administration of 99mTcHMPAO-labeled human mesenchymal stem cells after stroke: in vivo imaging and biodistribution. Cell Transplant 18:1369-1379. CrossRef Medline

Egashira Y, Sugitani S, Suzuki Y, Mishiro K, Tsuruma K, Shimazawa M, Yoshimura S, Iwama T, Hara H (2012) The conditioned medium of murine and human adipose-derived stem cells exerts neuroprotective effects against experimental stroke model. Brain Res 1461:87-95. CrossRef Medline

Faul M, Xu L, Wanld MM, Coronado VG (2010) Traumatic brain injury in the United States: emergency department visits, hospitalizations, and deaths. Atlanta GA: Centers for Disease Control and Prevention, National Center for Injury Prevention and Control.

Fox AH, Lamond AI (2010) Paraspeckles. Cold Spring Harbor Perspect Biol 2:a000687.

Fraser JK, Wulur I, Alfonso Z, Hedrick MH (2006) Fat tissue: an underappreciated source of stem cells for biotechnology. Trends Biotechnol 24: 150-154. CrossRef Medline

Galindo LT, Filippo TR, Semedo P, Ariza CB, Moreira CM, Camara NO, Porcionatto MA (2011) Mesenchymal stem cell therapy modulates the inflammatory response in experimental traumatic brain injury. Neurol Res Int 2011:564089. Medline

Glover LE, Tajiri N, Lau T, Kaneko Y, van Loveren H, Borlongan CV (2012) Immediate, but not delayed, microsurgical skull reconstruction exacerbates brain damage in experimental traumatic brain injury model. PLoS One 7:e33646. CrossRef Medline

Harting MT, Sloan LE, Jimenez F, Baumgartner J, Cox CS Jr (2009) Subacute neural stem cell therapy for traumatic brain injury. J Surg Res 153: 188-194. CrossRef Medline

Hawkins BE, Cowart JC, Parsley MA, Capra BA, Eidson KA, Hellmich HL, Dewitt DS, Prough DS (2013) Effects of trauma, hemorrhage and resuscitation in aged rats. Brain Res 1496:28-35. CrossRef Medline

Hayashi T, Kaneko Y, Yu S, Bae E, Stahl CE, Kawase T, van Loveren H, Sanberg PR, Borlongan CV (2009) Quantitative analyses of matrix metalloproteinase activity after traumatic brain injury in adult rats. Brain Res 1280:172-177. CrossRef Medline

Ibrahim AG, Raisman G, Li Y (2009) Permanent loss of fore-paw grasping requires complete deprivation of afferent input from a minimum of four dorsal roots of the rat brachial plexus. Exp Neurol 215:142-145. CrossRef Medline

Ikegame Y, Yamashita K, Hayashi S, Mizuno H, Tawada M, You F, Yamada K, Tanaka Y, Egashira Y, Nakashima S, Yoshimura S, Iwama T (2011) Comparison of mesenchymal stem cells from adipose tissue and bone marrow for ischemic stroke therapy. Cytotherapy 13:675-685. CrossRef Medline

Isacson O, Costantini L, Schumacher JM, Cicchetti F, Chung S, Kim K (2001) Cell implantation therapies for Parkinson's disease using neural stem, transgenic or xenogeneic donor cells. Parkinsonism Relat Disord 7:205-212. CrossRef Medline

Jang YY, Ye Z, Cheng L (2011) Molecular imaging and stem cell research. Mol Imaging 10:111-122. Medline 
Katsuda T, Tsuchiya R, Kosaka N, Yoshioka Y, Takagaki K, Oki K, Takeshita F, Sakai Y, Kuroda M, Ochiya T (2013) Human adipose tissue-derived mesenchymal stem cells secrete functional neprilysin-bound exosomes. Sci Rep 3:1197. CrossRef Medline

Kim WS, Park BS, Sung JH, Yang JM, Park SB, Kwak SJ, Park JS (2007) Wound healing effect of adipose-derived stem cells: a critical role of secretory factors on human dermal fibroblasts. J Dermatol Sci 48:15-24. CrossRef Medline

Lee P, Kim J, Williams R, Sandhir R, Gregory E, Brooks WM, Berman NE (2012) Effects of aging on blood brain barrier and matrix metalloproteases following controlled cortical impact in mice. Exp Neurol 234:5061. CrossRef Medline

Lefebvre JS, Maue AC, Eaton SM, Lanthier PA, Tighe M, Haynes L (2012) The aged microenvironment contributes to the age-related functional defects of CD4 T cells in mice. Aging Cell 11:732-740. CrossRef Medline

Lendeckel S, Jödicke A, Christophis P, Heidinger K, Wolff J, Fraser JK, Hedrick MH, Berthold L, Howaldt HP (2004) Autologous stem cells (adipose) and fibrin glue used to treat widespread traumatic calvarial defects: case report. J Craniomaxillofac Surg 32:370-373. CrossRef Medline

Li M, Li F, Luo C, Shan Y, Zhang L, Qian Z, Zhu G, Lin J, Feng H (2011) Immediate splenectomy decreases mortality and improves cognitive function of rats after severe traumatic brain injury. J Trauma 71:141-147. CrossRef Medline

Lindvall O, Brundin P, Widner H, Rehncrona S, Gustavii B, Frackowiak R, Leenders KL, Sawle G, Rothwell JC, Marsden CD, Bjorkland A (1990) Grafts of fetal dopamine neurons survive and improve motor function in Parkinson's disease. Science 247:574-577. CrossRef Medline

Liu YP, Lang BT, Baskaya MK, Dempsey RJ, Vemuganti R (2009) The potential of neural stem cells to repair stroke-induced brain damage. Acta Neuropathol 117:469-480. CrossRef Medline

Mahmood A, Lu D, Lu M, Chopp M (2003) Treatment of traumatic brain injury in adult rats with intravenous administration of human bone marrow stromal cells. Neurosurgery 53:697-702; discussion 702-703. CrossRef Medline

McDonald KG, Leach MR, Huang C, Wang C, Newberry RD (2011) Aging impacts isolated lymphoid follicle development and function. Immunity Ageing 8:1. CrossRef Medline

Mercer TR, Mattick JS (2013) Structure and function of long noncoding RNAs in epigenetic regulation. Nat Struct Mol Biol 20:300-307. CrossRef Medline

Michalet X, Pinaud FF, Bentolila LA, Tsay JM, Doose S, Li JJ, Sundaresan G, Wu AM, Gambhir SS, Weiss S (2005) Quantum dots for live cells, in vivo imaging, and diagnostics. Science 307:538-544. CrossRef Medline

Mizuno H, Tobita M, Uysal AC (2012) Concise review: adipose-derived stem cells as a novel tool for future regenerative medicine. Stem Cells 30:804-810. CrossRef Medline

Muraoka K, Shingo T, Yasuhara T, Kameda M, Yuen WJ, Uozumi T, Matsui T, Miyoshi Y, Date I (2008) Comparison of the therapeutic potential of adult and embryonic neural precursor cells in a rat model of Parkinson disease. J Neurosurg 108:149-159. CrossRef Medline

Naganuma T, Hirose T (2013) Paraspeckle formation during the biogenesis of long non-coding RNAs. RNA Biol 10(8). CrossRef

Nakagawa S, Hirose T (2012) Paraspeckle nuclear bodies-useful uselessness? Cell Mol Life Sci 69:3027-3036. CrossRef Medline

Paxinos G, Watson C (2007) The rat brain in stereotaxic coordinates, Ed 6. Amsterdam: Academic/Elsevier.

Qi JH, Ebrahem Q, Moore N, Murphy G, Claesson-Welsh L, Bond M, Baker A, Anand-Apte B (2003) A novel function for tissue inhibitor of metalloproteinases-3 (TIMP3): inhibition of angiogenesis by blockage of VEGF binding to VEGF receptor-2. Nat Med 9:407-415. CrossRef Medline

Ribeiro CA, Fraga JS, Grãos M, Neves NM, Reis RL, Gimble JM, Sousa N, Salgado AJ (2012) The secretome of stem cells isolated from the adipose tissue and Wharton jelly acts differently on central nervous system derived cell populations. Stem Cell Res Ther 3:18. CrossRef Medline

Sasaki YT, Ideue T, Sano M, Mituyama T, Hirose T (2009) MENepsilon/ beta noncoding RNAs are essential for structural integrity of nuclear paraspeckles. Proc Natl Acad Sci U S A 106:2525-2530. CrossRef Medline

Singer NG, Caplan AI (2011) Mesenchymal stem cells: mechanisms of inflammation. Annu Rev Pathol 6:457-478. CrossRef Medline

Solanto MV (1984) Neuropharmacological basis of stimulant drug action in attention deficit disorder with hyperactivity: a review and synthesis. Psychol Bull 95:387-409. CrossRef Medline

Spadaro PA, Bredy TW (2012) Emerging role of non-coding RNA in neural plasticity, cognitive function, and neuropsychiatric disorders. Front Genet 3:132. CrossRef Medline

Sugiyama T, Kuroda S, Osanai T, Shichinohe H, Kuge Y, Ito M, Kawabori M, Iwasaki Y (2011) Near-infrared fluorescence labeling allows noninvasive tracking of bone marrow stromal cells transplanted into rat infarct brain. Neurosurgery 68:1036-1047; discussion 1047. CrossRef Medline

Sun J, Zhou H, Deng Y, Zhang Y, Gu P, Ge S, Fan X (2012) Conditioned medium from bone marrow mesenchymal stem cells transiently retards osteoblast differentiation by downregulating runx2. Cells Tissues Organs 196:510-522. CrossRef Medline

Ushiki T, Kizaka-Kondoh S, Ashihara E, Tanaka S, Masuko M, Hirai H, Kimura S, Aizawa Y, Maekawa T, Hiraoka M (2010) Noninvasive tracking of donor cell homing by near-infrared fluorescence imaging shortly after bone marrow transplantation. PLoS One 5:e11114. CrossRef Medline

Vendrame M, Gemma C, Pennypacker KR, Bickford PC, Davis Sanberg C, Sanberg PR, Willing AE (2006) Cord blood rescues stroke-induced changes in splenocyte phenotype and function. Exp Neurol 199:191-200. CrossRef Medline

Vorhees CV, Williams MT (2006) Morris water maze: procedures for assessing spatial and related forms of learning and memory. Nat Protoc 1:848-858. CrossRef Medline

Walker PA, Shah SK, Harting MT, Cox CS Jr (2009) Progenitor cell therapies for traumatic brain injury: barriers and opportunities in translation. Dis Model Mech 2:23-38. CrossRef Medline

Walker PA, Shah SK, Jimenez F, Gerber MH, Xue H, Cutrone R, Hamilton JA, Mays RW, Deans R, Pati S, Dash PK, Cox CS Jr (2010) Intravenous multipotent adult progenitor cell therapy for traumatic brain injury: preserving the blood brain barrier via an interaction with splenocytes. Exp Neurol 225:341-352. CrossRef Medline

Wapinski O, Chang HY (2011) Long noncoding RNAs and human disease. Trends Cell Biol 21:354-361. CrossRef Medline

Xue S, Zhang HT, Zhang P, Luo J, Chen ZZ, Jang XD, Xu RX (2010) Functional endothelial progenitor cells derived from adipose tissue show beneficial effect on cell therapy of traumatic brain injury. Neurosci Lett 473: 186-191. CrossRef Medline

Yang M, Donaldson AE, Jiang Y, Iacovitti L (2003) Factors influencing the differentiation of dopaminergic traits in transplanted neural stem cells. Cell Mol Neurobiol 23:851-864. CrossRef Medline

Yao J, Jiang SL, Liu W, Liu C, Chen W, Sun L, Liu KY, Jia ZB, Li RK, Tian H (2012) Tissue inhibitor of matrix metalloproteinase-3 or vascular endothelial growth factor transfection of aged human mesenchymal stem cells enhances cell therapy after myocardial infarction. Rejuvenation Res 15: 495-506. CrossRef Medline

Yu S, Kaneko Y, Bae E, Stahl CE, Wang Y, van Loveren H, Sanberg PR, Borlongan CV (2009) Severity of controlled cortical impact traumatic brain injury in rats and mice dictates degree of behavioral deficits. Brain Res 1287:157-163. CrossRef Medline

Zhang B, Arun G, Mao YS, Lazar Z, Hung G, Bhattacharjee G, Xiao X, Booth CJ, Wu J, Zhang C, Spector DL (2012) The lncRNA Malatl is dispensable for mouse development but its transcription plays a cis-regulatory role in the adult. Cell Rep 2:111-123. CrossRef Medline 